\title{
Micron-scale laser-wire scanner for the KEK Accelerator Test Facility extraction line
}

\author{
Stewart T. Boogert, ${ }^{*}$ Grahame A. Blair, Gary Boorman, Alessio Bosco, Lawrence C. Deacon, ${ }^{\dagger}$ and Pavel Karataev \\ John Adams Institute at Royal Holloway, Egham, Surrey TW20 OEX, United Kingdom \\ Alexander Aryshev, Masafumi Fukuda, Nobihiro Terunuma, and Junji Urakawa \\ KEK, 1-1 Oho, Tsukuba, Ibaraki 305-0801, Japan

\begin{abstract}
Laura Corner, Nicolas Delerue, Brian Foster, David Howell, Myriam Newman, ${ }^{\ddagger}$ Rohan Senanayake, Roman Walczak, and Fred Ganaway ${ }^{\S}$

John Adams Institute at Oxford University, Nuclear and Astrophysics Laboratory, Keble Road, Oxford OX1 3RH, United Kingdom
\end{abstract} (Received 16 July 2010; published 6 December 2010)

\begin{abstract}
A laser-wire transverse electron beam size measurement system has been constructed and operated at the Accelerator Test Facility (ATF) extraction line at KEK. The construction of the system is described in detail along with the environment of the ATF related to the laser wire. A special set of electron beam optics was developed to generate an approximately $1 \mu \mathrm{m}$ vertical focus at the laser-wire location. The results of our operation at the ATF extraction line are presented, where a minimum rms electron beam size of $4.8 \pm 0.3 \mu \mathrm{m}$ was measured, and smaller electron beam sizes can be measured by developing the method further. The beam size at the laser-wire location was changed using quadrupoles and the resulting electron beam size measured, and vertical emittance extracted.
\end{abstract}

DOI: 10.1103/PhysRevSTAB.13.122801

PACS numbers: 41.85.Ew, 29.20.Ej

\section{INTRODUCTION}

Electron-positron accelerators such as a linear collider and other low emittance accelerator facilities, like light sources, will generate beams with transverse beam sizes of the order of $1 \mu \mathrm{m}$ [1]. The normal techniques of beam size measurement, for example, wire scanners and screens which intersect the charged particle beam, are not applicable when the bunch charge density is sufficiently large to damage the material placed in the beam. In these scenarios noninvasive techniques must be used such as optical diffraction radiation [2] or methods based on laser interactions with electron beams. A review of beam size measurement of electron beams can be found in [3]. A number of Compton scattering diagnostics have already been developed for beam size measurement, such as the Stanford Linear Collider (SLC) laser wire [4,5], ATF damping ring laser wire [6], and PETRA laser wire [7]. A method for measuring submicron beams using a laser interference pattern has also been developed [8]. A related technology for application at proton machines is a laser wire based on hydrogen ion neutralization as opposed to Compton scattering [9].

The aim of the ATF extraction line laser-wire project is to develop a system capable of reliably measuring an electron beam of the order of $1 \mu \mathrm{m}$ in vertical transverse

\footnotetext{
*sboogert@pp.rhul.ac.uk

†deacon@pp.rhul.ac.uk

${ }^{\ddagger}$ Now at the European Organization for Nuclear Research, CERN CH-1211, Genève 23, Switzerland.

${ }^{\S}$ Now at Queen Mary, University of London, Physics Department, Mile End Road, London E1 4NS, UK.
}

size [10]. This paper describes our prototype hardware system in which we have tested various optical focusing and operation schemes. We report on the operation with the extraction line laser wire, including a custom made short focal length final focus lens, interaction chamber mover system, and knife edge vacuum manipulator, and on results obtained using this system, including the ATF extracted beam emittance measurement.

\section{A. Laser wire}

A laser wire uses a focused laser pulse which interacts with the electron beam via Compton scattering. The laser photons are scattered to higher energies, almost parallel to the incident electron beam, while the scattered electrons are reduced in energy. Moving the laser focus across the electron beam while monitoring the rate of Compton scattered photons provides information on the electron beam transverse charge density. A key requirement is knowledge of the laser beam radius at the collision point. We briefly review Gaussian laser beams [11] as relevant for the ATF laser wire. The laser beam must be focused so that the laser beam is smaller than or comparable to the electron beam. For a Gaussian laser beam, in the absence of spherical aberrations, the beam radius at focus, created by a simple converging lens of focal length $f$, assuming an infinite lens aperture and plane wave illumination is given by

$$
W_{0}=\frac{M^{2} f \lambda}{W_{1} \pi}
$$

where $W_{0}$ is the laser beam radius at the laser waist, $W_{1}$ is the laser beam size incident on the lens, $\lambda$ is the laser 
wavelength, and $M^{2}$ is a measure of the transverse mode quality of the laser. For a Gaussian beam the radius $W$ is defined as the distance between the peak of the intensity distribution and the point at which it reaches $1 / e^{2}$ of its peak value. The radius of such a beam propagates according to

$$
W(z)=W_{0}\left[1+\left(\frac{M^{2} \lambda}{\pi W_{0}^{2}}\left(z-z_{0}\right)\right)\right]^{1 / 2},
$$

where $z$ is the distance along the optical axis and $z_{0}$ is the waist location. The Rayleigh range is defined as the propagation distance over which the beam size grows to $\sqrt{2}$ of the focused value $W_{0}$ and is given by

$$
z_{R}=\frac{\pi W_{0}^{2}}{M^{2} \lambda}
$$

In the presence of aberrations, Eqs. (1) and (3) are modified and in general optical simulation or measurement of the laser propagation is typically required to determine the smallest achievable focused spot size and the beam size near the focus. In the presence of spherical aberrations the $M^{2}$ used in Eqs. (1) and (3) is modified according to the following equation $[12,13]$ :

$$
M^{2}=\left[\left(M_{0}^{2}\right)^{2}+\left(M_{q}^{2}\right)^{2}\right]^{1 / 2},
$$

where $M_{0}^{2}$ is the initial beam quality factor and $M_{q}^{2}$ is due to a lens producing spherical aberrations.

The laser focus is moved transversely by adjusting the incoming angle of the laser beam on the focusing lens. Using simple geometric optics, the movement of the focus $\left(\delta y_{L}\right)$ that can be produced by changing the angle of the laser beam on the focusing lens $\left(\delta \theta_{y}\right)$ is given by

$$
\delta y_{L}=f \delta \theta_{y} .
$$

There are a large number of devices which can rapidly deflect a laser beam such as piezoelectric stacks, acoustoelectric and electro-optic scanners. However, the development of such scanners that can maintain the beam quality, sustain high laser power densities, and can scan beams over $\mu \mathrm{rad}$ at frequencies of the order of $100 \mathrm{kHz}$ [14] is an active research topic.

Another method of scanning the laser beam is to change the position of the lens itself. Using this method, a change in transverse lens position corresponds to a change in laser focus position.

\section{B. Compton scattering}

The average total rate of Compton photons $\left\langle N_{\gamma}\right\rangle$ generated by a laser pulse colliding with an electron pulse is given by [3]

$$
\left\langle N_{\gamma}\right\rangle=N_{b} P_{L} \frac{\sigma_{C} \lambda}{c h} \int_{x} \int_{y} \int_{z} \rho_{e}(x, y, z) \rho_{L}(x, y, z) d x d y d z,
$$

where $\sigma_{C}$ is the Compton cross section, $N_{b}$ is the bunch population, $P_{L}$ is the laser pulse power, $\lambda$ the laser wavelength, and $\rho_{e}$ and $\rho_{L}$ are the electron and photon number densities, respectively. This also assumes that the laser pulse duration is significantly longer than that of the electron pulse. In the case where both the laser energy and electron charge density distributions are Gaussian, and where the laser Rayleigh range is significantly longer than the horizontal beam size, Eq. (6) can be simplified by performing the integral to give [3]

$$
\left\langle N_{\gamma}\right\rangle=N_{b} P_{L} \frac{\sigma_{C} \lambda}{c h} \frac{1}{\sqrt{2 \pi} \sigma_{s}} \exp \left(-\frac{\delta y^{2}}{2 \sigma_{s}^{2}}\right),
$$

where $\delta y=y_{L}-y_{e}$ is the vertical position difference between electron and laser beam centers (Fig. 1) and $\sigma_{s}$ is the quadrature sum of the vertical laser and electron beam sizes $\left(\sigma_{s}^{2}=\sigma_{L 0}^{2}+\sigma_{e}^{2}\right)$ at the laser-wire interaction point (LWIP). Then by measuring the modulation of the Compton rate $\left(N_{\gamma}\right)$ as a function of relative displacement $(\delta y)$, the quadrature beam size $\left(\sigma_{s}\right)$ can be extracted. $\sigma_{L 0}$ is the smallest vertical laser beam size at the LWIP. Provided this is known, or is small enough that it is negligible when added in quadrature with the electron beam size, the vertical electron beam size $\left(\sigma_{e}\right)$ can be extracted from $\sigma_{s}$. A similar procedure could be carried out in the horizontal axis.

The laser photons are typically scattered to larger energies (inverse Compton scattering) and the spectrum of scattered photons is given by [3]

$$
\begin{aligned}
\frac{d \sigma / \sigma_{0}}{d w}= & \frac{3}{8 \epsilon}\left[\frac{1}{1-w}\right]+1-w+\left[\frac{w}{\epsilon(1-w)}\right]^{2} \\
& -\frac{2 w}{\epsilon(1-w)},
\end{aligned}
$$

where $\sigma$ is the cross section, $\sigma_{0}$ is the Thomson scattering cross section, and $\epsilon$ is the normalized energy of the laser photon in the electron rest frame, $\gamma h c /\left(\lambda m_{e}\right)$. Here $\gamma$ is the relativistic factor and $w=E_{\gamma} / E$, where $E_{\gamma}$ is the scattered photon energy and $E$ is the electron energy. The maximum energy of scattered photons is given by

$$
E_{\gamma, \max }=2 E \epsilon /(1+2 \epsilon) \text {. }
$$

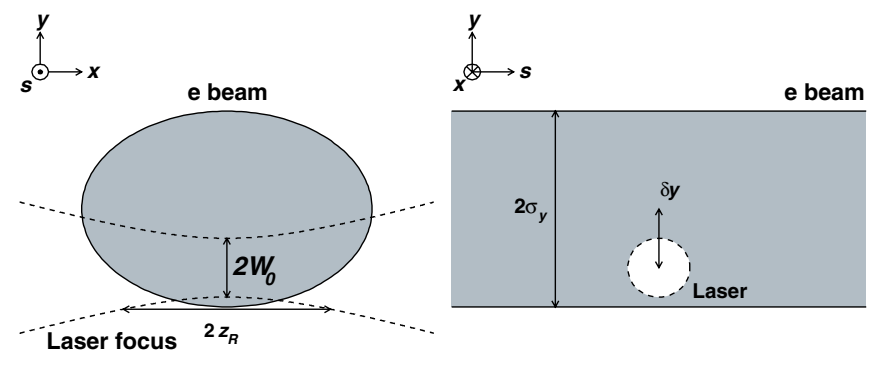

FIG. 1. Geometry of the laser and electron beam interaction. 
TABLE I. ATF parameters beam parameters at the beginning of the extraction line.

\begin{tabular}{lc}
\hline \hline Parameter & Value \\
\hline Beam energy & $1.28 \mathrm{GeV}$ \\
Fractional energy spread & $6.4 \times 10^{-4}$ \\
Bunch charge & $1 \times 10^{10}$ electrons \\
rf frequency & $714 \mathrm{MHz}$ \\
Extraction frequency & 1.56 to $6.24 \mathrm{~Hz}$ \\
Bunch length & $\leq 30 \mathrm{ps}$ \\
Vertical emittance $\epsilon_{y}$ & $5 \times 10^{-11} \mathrm{mrad}$ \\
Horizontal emittance $\epsilon_{x}$ & $1.6 \times 10^{-9} \mathrm{mrad}$ \\
\hline \hline
\end{tabular}

\section{ATF AND ELECTRON BEAM OPTICS}

The ATF is a test accelerator to investigate the radiation damping of an electron beam for injection into the linac of the ILC [15]. Table I shows the major parameters of the ATF. The ATF consists of a radio frequency (rf) laser injector system and S-band linac which accelerates the electron beam to an energy of $1.28 \mathrm{GeV}$. The beam is injected into the ATF damping ring where it is stored for typically $0.5 \times 10^{6}$ revolutions, until the beam reaches the equilibrium vertical emittance of $5 \times 10^{-11} \mathrm{mrad}$ and horizontal emittance $1.6 \times 10^{-9} \mathrm{mrad}$. The beam is extracted using a double kicker and septum magnet system into the extraction line that is used for testing advanced diagnostics and to safely transport the beam to the dump, as shown in Fig. 2.

A special set of optics was developed to generate a small beam size at the laser-wire location. The Methodical Accelerator Design program (MAD) [16-18] was used to develop a simulation of the ATF extraction line. The results are shown in Fig. 3. The laser-wire interaction chamber was located in between two ATF extraction line dipoles at $s=21 \mathrm{~m}$. The lattice was designed such that the first order dispersion could be corrected in the diagnostics section of the extraction line, downstream of dipole BH2X.1. The ATF extraction line optics did not allow the horizontal dispersion function to be nulled with zero gradient in the region around the LWIP. However, the dispersion sign

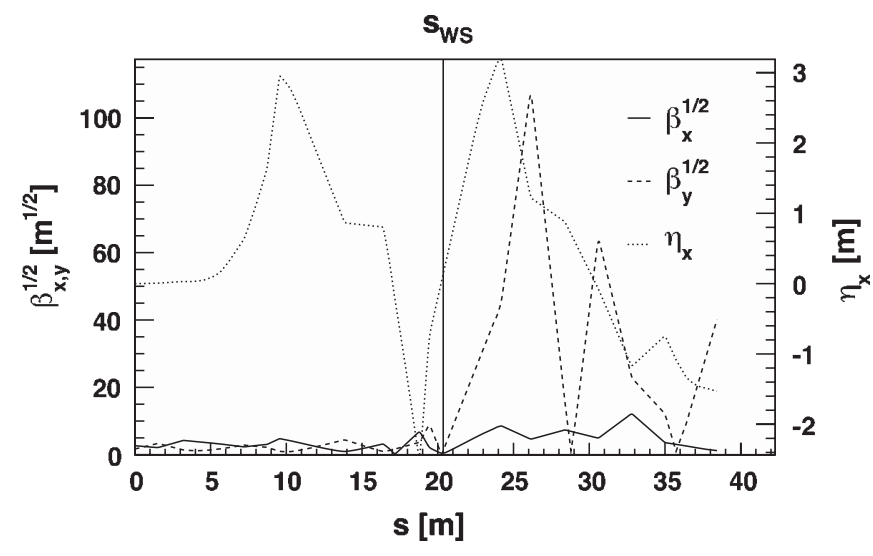

FIG. 3. Horizontal and vertical betatron $\left(\beta_{x}, \beta_{y}\right)$ and horizontal dispersion $\left(\eta_{x}\right)$ functions for the ATF extraction line. The vertical line marks the location of the wire scanner $\left(\mathrm{S}_{\mathrm{WS}}\right)$, close to the LWIP.

could be changed from negative to positive around the LWIP, creating a null point.

\section{A. Verification of electron beam optics}

The horizontal dispersion zero point and the laser-wire IP (LWIP) beam size were verified by carrying out both horizontal and vertical dispersion $\left(\eta_{x}, \eta_{y}\right)$ measurements using two calibrated beam position monitors (BPMs) attached to the interaction chamber upstream and downstream of the LWIP and the standard ATF BPMs (see Fig. 4). These dispersion measurements were performed concurrently with the laser-wire collision tests presented in Sec. V. Emittance measurements were performed in the preceding weeks with the quadrupole scan method [19] using a downstream wire scanner and quadrupole in the dispersion free region.

To measure the dispersion, the beam energy was varied by ramping the radio frequency (rf) cavities in the damping ring, the beam position was plotted against $\delta E$, and the tangent was extracted from the resulting parabola. The $\eta_{y}$ and $\eta_{x}$ measured using this technique along with the $\eta_{x}$ calculated from MAD is plotted in Fig. 4. Although the

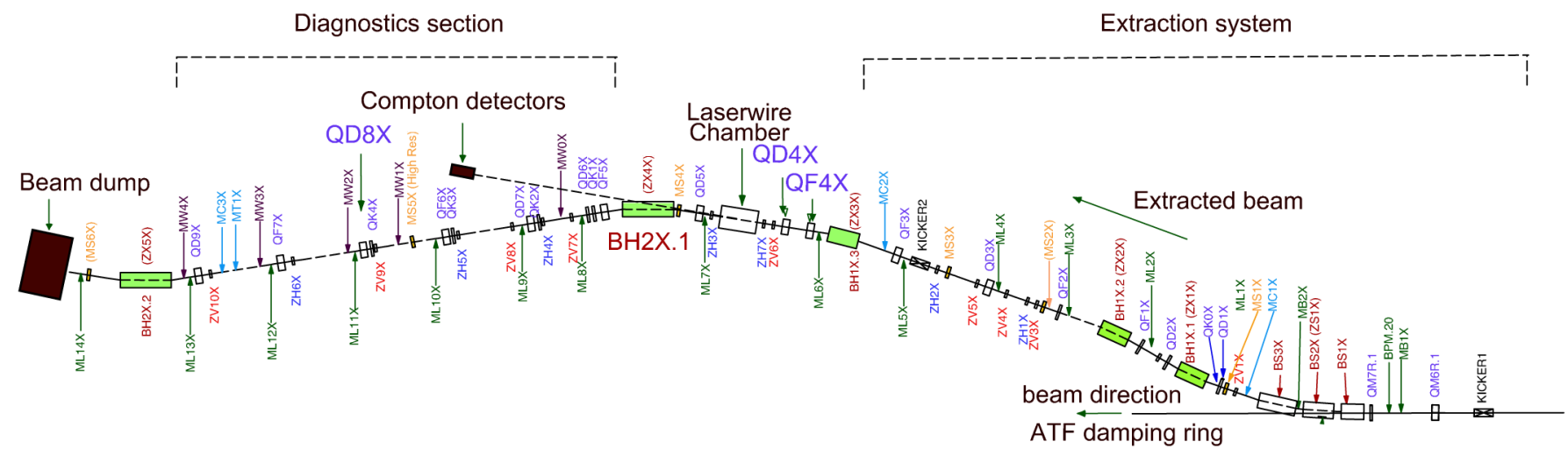

FIG. 2. ATF extraction line from kicker to dump. 

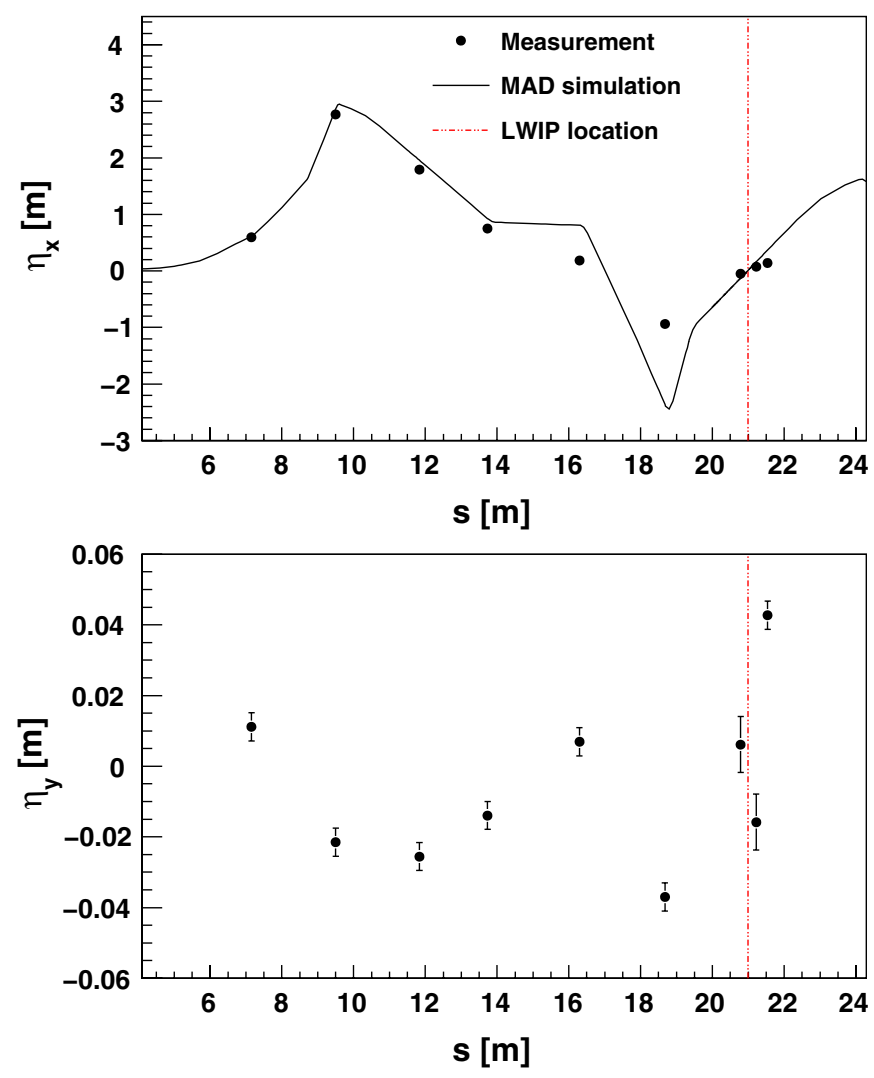

FIG. 4. ATF extraction line measured and simulated dispersion. The upstream quadrupole focusing strengths were $l k_{\mathrm{QD} 4 \mathrm{X}}=-1.52 \mathrm{~m}^{-1}$ and $l k_{\mathrm{QF} 4 \mathrm{X}}=1.58 \mathrm{~m}^{-1}$, where $l k=$ $\frac{l}{B \rho} \frac{d B_{z}}{d x}$, where $l$ is the length of the quadrupole, $\frac{1}{B \rho}$ is the magnetic rigidity, and $\frac{d B_{z}}{d x}$ is the field gradient. $s=0$ corresponds to the beginning of the extraction line. The vertical dotted line indicates the laser-wire location.

vertical dispersion should nominally be zero, Fig. 4 shows there was some residual dispersion. The dispersion at the LWIP was measured as $\eta_{x}=(-3.2 \pm 0.1) \times 10^{-1} \mathrm{~m}$ and $\eta_{y}=(-3.9 \pm 0.8) \times 10^{-3} \mathrm{~m}$. This was done by taking beam position measurements from two BPMs, one upstream and one downstream of the LWIP, and assuming a linear dispersion function between these two points. This is a reasonable assumption because there are no magnetic fields in this region. The error in $\eta$ includes a statistical error and a systematic error associated with the error in the position of the LWIP. The dispersion crosses zero $9 \mathrm{~cm}$ upstream of the LWIP. Using $\frac{\delta E}{E}=7.8 \times 10^{-4}$ [20], the beam size due to dispersion $\sigma_{\eta}$ is $3.03 \pm 0.66 \mu \mathrm{m}$.

The emittance was measured by performing a quadrupole scan with quadrupole QD8X and wire scanner MW3X, $2.52 \mathrm{~m}$ downstream from QD8X as $207 \pm 2 \mathrm{pm}$. The minimum $\beta$ function, calculated by MAD, is $0.008 \mathrm{~m}$. Therefore the vertical electron beam size at the LWIP, without dispersion, is expected to be $\sigma_{e, \beta}=1.28 \mu \mathrm{m}$, and including dispersion is $\sigma_{e}=\sqrt{\sigma_{e, \beta}^{2}+\sigma_{e, \eta}^{2}}=3.29 \mu \mathrm{m}$.

\section{HARDWARE INSTALLATION}

A schematic of the major beam line components of the laser-wire system is shown in Fig. 5. This section describes the main elements of the laser-wire system. The key elements are the high power pulsed laser, laser focusing optics, interaction region chamber, and Compton photon detectors.

\section{A. Laser system}

The laser system was located above the beam line experiment, on the shielding blocks of the ATF, in a temperature controlled room. The laser consists of three main components: a passively mode locked seed laser, a regenerative amplifier (RGA), and a linear amplifier. A neodymium vanadate (Nd:VAN) seed laser produces $20 \mathrm{ps}$ long, $1064 \mathrm{~nm}$ pulses at $357 \mathrm{MHz}$ with an average output power of $600 \mathrm{~mW}$. It is frequency locked to a $357 \mathrm{MHz}$ signal derived from the ATF rf system. Two Pockels cells are used to pick pulses at the ATF extraction frequency of $1.56 \mathrm{~Hz}$, which are injected into the flash lamp pumped neodymium yttrium aluminum garnet (Nd:YAG) RGA where they are stretched to $\sim 150 \mathrm{ps}$ and amplified to $15 \mathrm{~mJ}$. The Pockels cells are triggered from a timing signal from the ATF extraction kicker. These pulses are then amplified in two single pass flash lamp pumped Nd:YAG amplifiers to a typical energy of $900 \mathrm{~mJ}$. The pulses are frequency doubled in a potassium dideuterium phosphate (KD*P) crystal to $532 \mathrm{~nm}$ with a pulse energy of $400 \mathrm{~mJ}$, and separated from the remaining $1064 \mathrm{~nm}$ radiation using a dichroic mirror. This frequency doubling has the advantage that, in accordance with Eq. (1), a shorter wavelength gives a smaller spot size at the focus, and visible light is easier to align at the LWIP. To achieve the smallest (diffraction limited) spot size at the LWIP requires both that the focusing optics are of an extremely high quality and that the spatial mode of the laser beam is close to a perfect $\mathrm{TEM}_{00}$ mode, i.e., an $M_{0}^{2}$ close to 1 .

The laser pulses were transported $9 \mathrm{~m}$ to a $3 \times 1 \mathrm{~m}$ optical table placed parallel with the ATF extraction line, used for laser beam diagnostics and alignment. Two

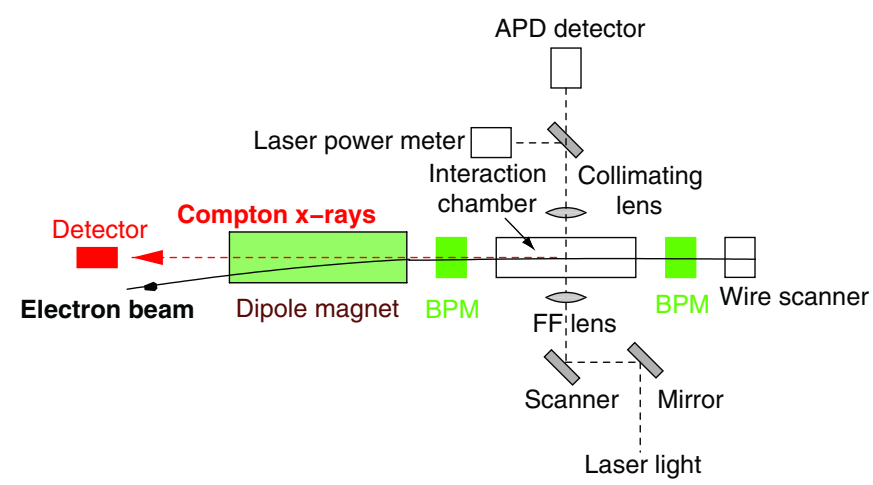

FIG. 5. Schematic of the laser-wire interaction region installation. 


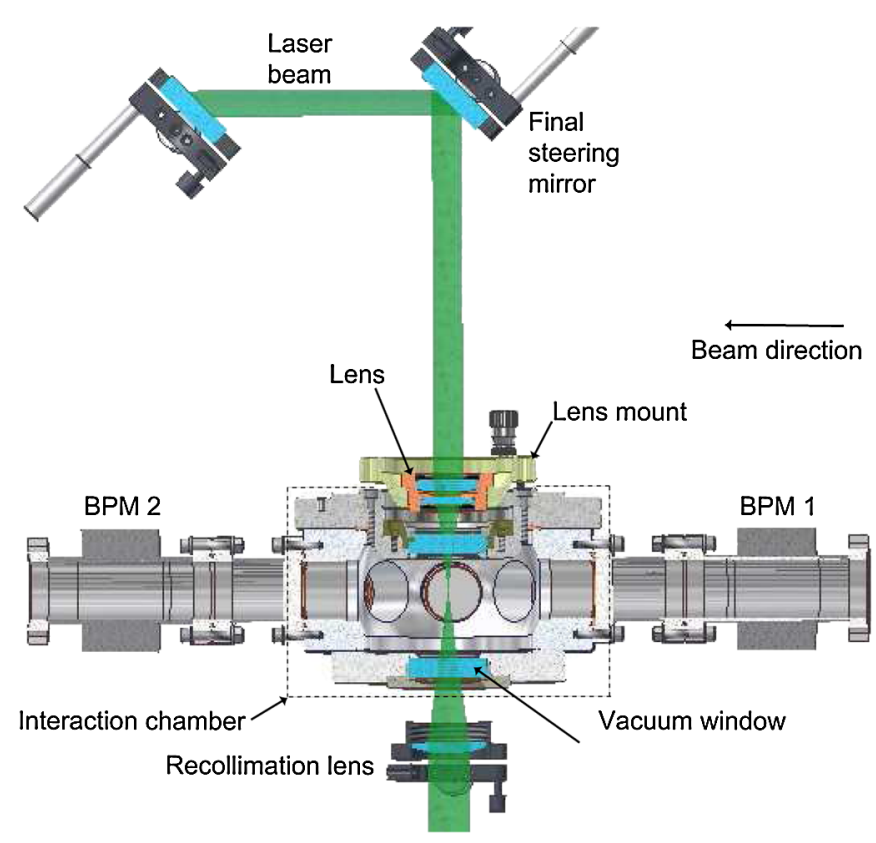

FIG. 6. Horizontal section of the laser-wire system, showing the interaction chamber, focusing lens, collimation lens, and the final scanning mirror.

smaller optical breadboards were placed on either side of the beam line. Two final mirrors steered the laser light onto the focusing lens used to create the focus inside the interaction chamber, as shown in Fig. 6.

\section{B. Final focus lens}

The laser was focused at the interaction point by a custom doublet lens of focal length $56.6 \mathrm{~mm}$ (Fig. 7). The lens consists of three elements, the first two elements with curved surfaces and then a vacuum window, which is an integral part of the lens design. The first curved surface is aspheric to correct for spherical aberrations. All of the optical elements were made of fused silica to withstand both high laser power and a high radiation environment. The lens has a high damage threshold antireflective coating to prevent the formation of ghosts within the lens, which could destroy it. The lens design parameters are shown in Tables II and III. Equation (10) is the equation describing an even asphere surface, like the first surface of the final

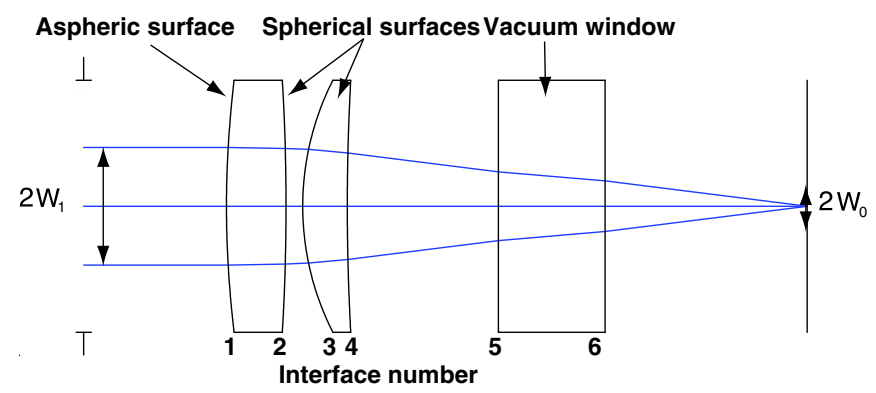

FIG. 7. Diagram of the final focus lens. focus lens (material interface number 1 in Tables II and III and Fig. 7):

$$
z=\frac{c r^{2}}{1+\sqrt{1-(1+k) c^{2} r^{2}}}+\alpha_{1} r^{2}+\alpha_{2} r^{4}+\alpha_{3} r^{6}+\cdots
$$

Here $z$ is the distance of the surface in the direction of the optical axis, $c=\frac{1}{R}$, where $R$ is the radius of curvature, $k$ is the conic constant, and the $\alpha_{i}$ are the higher order terms, given in Tables II and III. The tilts of the final two mirrors were controlled by two remote controlled DC-servo linear actuators. Additionally, the entire interaction chamber, to which the lens was affixed, could be moved horizontally $(x)$ and vertically $(y)$, so the lens position both vertically and along the laser beam propagation axis could be adjusted. The laser focus could be moved by either moving the interaction chamber or changing the tilt of the mirrors. The focusing lens was also mounted on a manually actuated translation and tilt system, to align the lens with respect to the chamber center. After the laser beam exits the interaction chamber another lens was used to recollimate the divergent light from the LWIP. A further lens was used to bring it to an acceptable size onto a power meter. This recollimation lens was also remotely movable, so that both the pre-LWIP and the post-LWIP lenses could be simultaneously moved in order to keep the same beam size on the laser power meter and other detectors.

\section{Lens simulation}

A simulation of the lens and interaction chamber window setup was carried out using ZEMAX [21] to find the $M_{q}^{2}$ of the lens [Eq. (4)] as a function of input beam size assuming an input laser with an $M_{0}^{2}=1$. ZEMAX uses a Fourier transform based beam propagation model called "physical optics propagation" (POP) to compute laser intensity on different optical surfaces. The intensity distribution is calculated on a two-dimensional rectangular grid and the laser beam size at focus computed by calculating the second moment of the ZEMAX simulated intensity distribution. The laser beam profiling systems used to measure the laser beam sizes apply a cut to remove the background of $0.3 \%$ of the peak projected intensity, and this was also applied to the ZEMAX computed intensity profiles. It was found that the calculated focused beam profile was slightly dependent on the grid size used. This is illustrated in Fig. 8, which shows the input laser beam size $W_{1}$ plotted against the focused spot size $W_{0}$. The dashed and dotted lines show the minimum and maximum values of $W_{0}$ for each value of $W_{1}$ calculated using the different grid sizes $(128,256,512,1024$, and 2048 pixels squared). The data points were taken using a beam profiler [22] to measure the focused spot size after the lens using a continuous wave (CW) laser with an $M_{0}^{2}$ of 1 and are an excellent fit to the simulation. The $M_{q}^{2}$ of the lens can be 
TABLE II. Custom laser-wire lens design parameters. "Thickness" is the distance from the previous surface along the optical axis. "Radius" is the radius of curvature of the surface. Additional parameters for interface number 1 are shown in Table III.

\begin{tabular}{|c|c|c|c|c|c|}
\hline Interface number & Shape & Radius [mm] & Thickness [mm] & From & To \\
\hline 1 & Even asphere & 117.126106 & 7.093310 & Air & Silica \\
\hline 2 & Spherical & -250.070725 & 1.987140 & Silica & Air \\
\hline 3 & Spherical & 33.118324 & 5.309160 & Air & Silica \\
\hline 4 & Spherical & 274.998672 & 17.985135 & Silica & Air \\
\hline 5 & Spherical & Infinity & 12.700000 & Air & Silica \\
\hline 6 & Spherical & Infinity & 24.075710 & Silica & Vacuum \\
\hline
\end{tabular}

TABLE III. Parameters in Eq. (10) describing the surface shape of interface number 1 of the custom laser-wire lens.

\begin{tabular}{lccc}
\hline \hline$k$ & $\alpha_{1}$ & $\alpha_{2}$ & $\alpha_{3}$ \\
\hline-14.455280 & 0 & $2.160486 \times 10^{-7}$ & $-7.467086 \times 10^{-10}$ \\
\hline \hline
\end{tabular}

calculated from Eq. (1) using the ZEMAX values of $W_{1}$ and $W_{0}$ and the results are shown in Fig. 9. The $M_{q}^{2}$ of the lens is due to spherical aberrations when it is overfilled and goes as the fourth power of input beam size $[12,13]$ and therefore the simulated $M_{q}^{2}$ were fitted to a fourth order polynomial.

The $M_{q}^{2}$ of the final focus lens can be combined with the measured laser $M_{0}^{2}$ using Eq. (4) to determine the focused spot size of the laser at the LWIP. It is clear from Fig. 8 that for the smallest focus the best input beam size $W_{1}$ is between 5 and $7 \mathrm{~mm}$ and should produce a $W_{0}$ of 1.76-1.79 $\mu \mathrm{m}$, provided the laser has $M_{0}^{2}=1$.

\section{Interaction chamber}

A custom interaction chamber was machined from a single block of vacuum grade stainless steel (T316LN

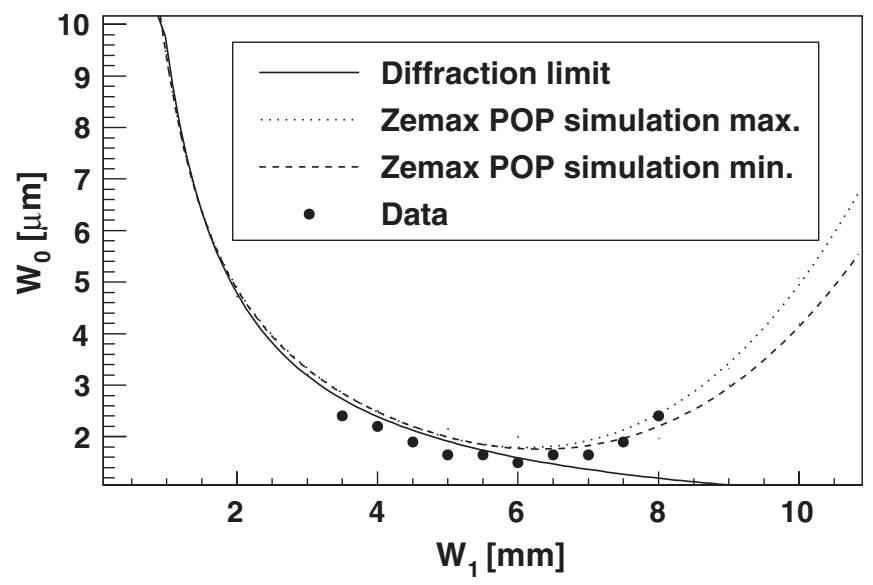

FIG. 8. Results of ZEMAX simulation of the $W_{0}$ of the custom lens for different input beam sizes and grid sizes. The minimum and maximum of the model are shown. The input laser has $M_{0}^{2}=1$. electroslag refined, cross forged, and heat treated to stress relive prior to machining). The interaction chamber is shown in Figs. 6 and 10. As short focal length optics were used, the chamber was designed to minimize the distance between the vacuum window and chamber center, while maintaining an electron beam stay clear of $15 \mathrm{~mm}$. This resulted in a thin octagonal chamber with large flanges on the laser entrance and exit faces to allow future reentrant window mounts to be employed.

A special indium vacuum seal was developed to mount the laser windows while minimizing the possibility of distorting the window, shown in detail in Fig. 11. The sealing surface is around the circumference of the optic to avoid uneven compressive forces distorting the optical surface. This solution is vital to minimize wavefront distortion.

To assist with the procedure of obtaining temporal and spatial overlap between the electron and laser beams (see Sec. V), a screen/knife edge was mounted on the end of a 2 inch travel vacuum translator. The knife edge vacuum translator has four directions of movement: one rotation axis with $0.1^{\circ}$ precision and three translation axes with micron precision. The insertion/retraction and rotation axes are controlled using stepper motors and can be

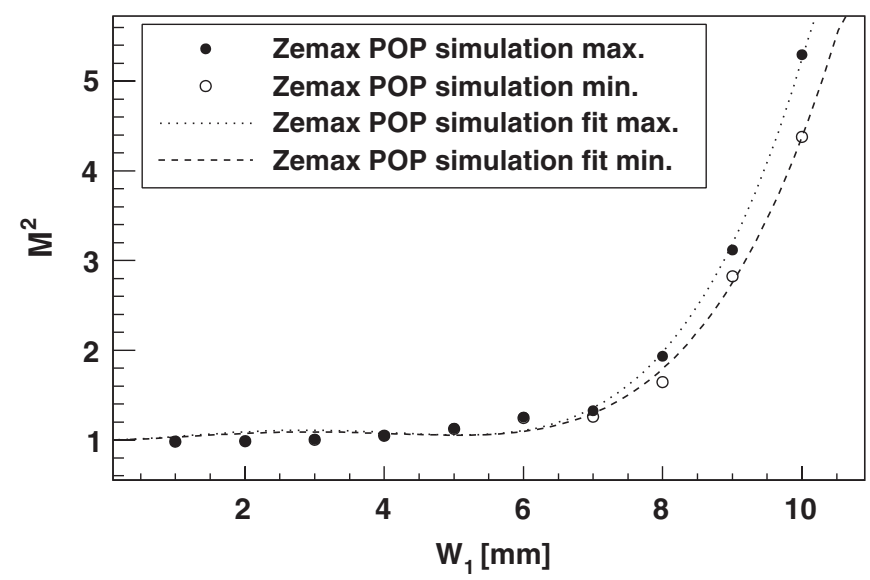

FIG. 9. Results of ZEMAX simulation of the $M^{2}$ after propagating through the custom lens of a laser beam with input $M_{0}^{2}=1$ for different input beam sizes and grid sizes. The minimum and maximum of the model are shown. 


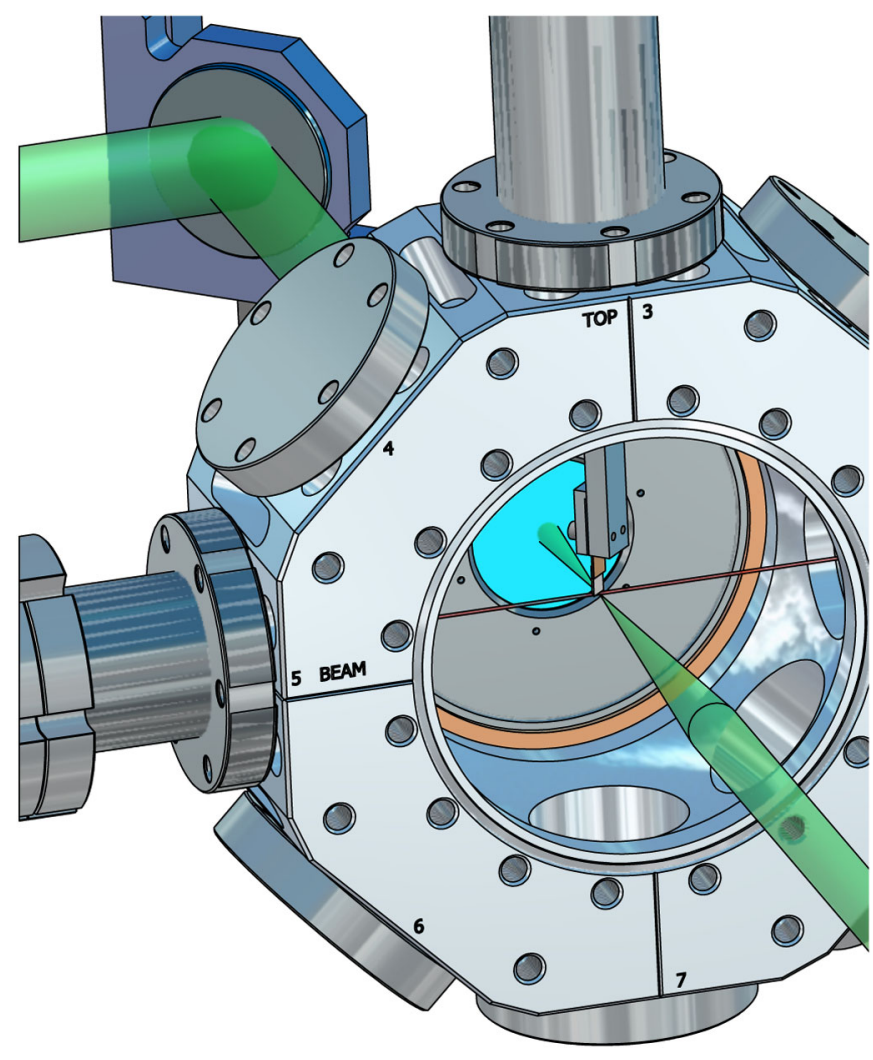

FIG. 10. View of the interaction chamber with the laser exit side flange removed, showing the $45^{\circ}$ screen/knife edge.

operated remotely as part of the data acquisition and control system for use during an electron beam collision search. The other two axes are controlled manually for prior laser focus positioning within the interaction chamber (Fig. 10). The knife edge was fabricated from a $300 \mu \mathrm{m}$ thick single crystal of silicon using etch and mask and then coated with gold [2].

Cross-secton D $(4: 1)$

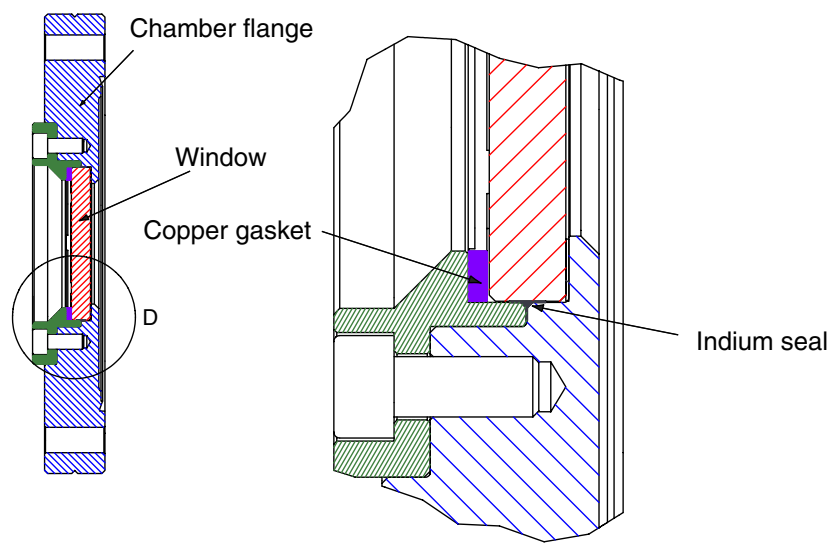

FIG. 11. Cross section of the window clamping and sealing arrangements.
The laser was aligned in the center of and perpendicular to the lens surface using the final two mirrors. The vertical position of the laser beam was changed by moving the interaction chamber vertically.

\section{Compton photon detectors}

A laser waist vertical size $\left(\sigma_{L 0}\right)$ of $5 \mu \mathrm{m}$ and peak power of $150 \mathrm{MW}$ incident on an electron beam of charge $1 \times 10^{10}$ electrons with a vertical beam size $\left(\sigma_{e}\right)$ of $1 \mu \mathrm{m}$ generates $6.8 \times 10^{4}$ Compton scattered $\gamma$-ray photons. The maximum energy of these photons, using Eq. (9), is 28.6 MeV. The total energy of the scattered photons per collision is approximately $975 \mathrm{GeV}$. The scattered photons exited the extraction line via a $1 \mathrm{~mm}$ thick aluminum window and traveled through $7.7 \mathrm{~m}$ of air to the first detector system. Two types of detector were used to measure the Compton $\gamma$ rays. The primary detector was an Aerogel Cherenkov detector. First the $\gamma$ rays were converted into electron-positron pairs using $7.35 \mathrm{~mm}$ of lead. Then the electron-positron pairs radiated Cherenkov radiation in $5.5 \mathrm{~cm}$ of Matsushita Denshi SP-15 Aerogel, with an area in the beam direction of $10 \times 10 \mathrm{~cm}$. The refractive index of the Aerogel is 1.015, with a Cherenkov threshold of $2.983 \mathrm{MeV}$. The Cherenkov light was guided down to a photomultiplier tube (PMT) at floor level which prevents direct beam induced backgrounds generating a signal in the PMT. A BDSIM [23] simulation from the LWIP to the detector was developed and samples of 1000 photons from the laser wire were simulated. In this simulation $10 \%$ of the photons were below the Cherenkov threshold, $1 \%$ were converted to $e^{+} e^{-}$by the aluminum window and finally the number of electrons plus the number of positrons above the Aerogel Cherenkov threshold entering the Aerogel itself was $14 \%$ of the number of Compton scattered photons (Fig. 12).

The second detector was a calorimeter composed of a single $(110 \mathrm{~mm} \times 120 \mathrm{~mm} \times 360 \mathrm{~mm})$ lead glass crystal coupled directly to a photomultiplier tube. This detector was placed directly behind the Aerogel detector and measured all the photons that were not converted by the thin lead plate. This was approximately $85 \%$ of the photons produced at the LWIP. This detector directly measured the total energy of the Compton scattered photons.

These two methods are complementary, the Cherenkov detector counting the photons generated and the calorimeter measuring the total energy. The charged particle background environment in the ATF was difficult to control and a great deal of beam time was required to reduce the backgrounds in the two detectors while preserving a small beam size at the LWIP.

\section{E. Data acquisition}

The data acquisition (DAQ) system for the laser wire was based upon multiple small executable programs written either in $\mathrm{C}++$ or $\mathrm{LABVIEW}^{\mathrm{TM}}$. A central data acquisition 


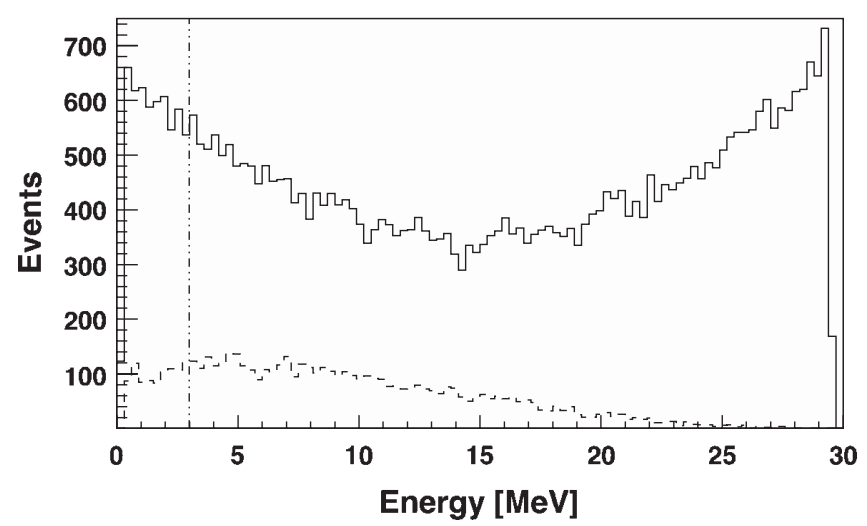

FIG. 12. The BDSIM [23] simulation results. Solid line, energy spectrum of Compton photons produced from the laser wire. Dashed line, the energy spectrum of the electrons or positrons from the electron or positron pairs entering the Aerogel. The vertical line indicates the Cherenkov threshold of the aerogel.

system communicated to each program via a short messaging protocol based on TCP/IP. This enabled the varied components of such a system (accelerator, optical devices, digital to analogue converter) to communicate and distribute data effectively. The ATF repetition frequency is sufficiently low that the latency in network communication does not lead to desynchronization of recorded data. The whole DAQ system was clocked via a trigger pulse which was generated from a signal from the ATF extraction kicker when it fired. This trigger pulse was used to gate all the digitizers and was monitored in software to notify the entire DAQ system of beam arrival in the extraction line. After the laser was optimized and collisions between the laser and electron beam were detected, the whole experiment was conducted in the ATF control room, from where remote control of the entire system was possible.

\section{LASER PROPAGATION MEASUREMENTS}

As discussed in Sec. I, to find the electron beam size from a laser-wire measurement it is necessary to know the laser spot size at the LWIP. In principle, it is possible to measure the laser beam size of the pulsed laser at focus using the knife edge and appropriate laser power detector. This proved impractical for numerous reasons. First, the lowest power output from the RGA could still easily damage the knife edge and attenuation would introduce a systematic error. Second, the knife edge could only be moved with micron precision, yielding relatively few measurements over a laser beam profile of a few microns. An imaging screen with magnifying optics and camera could have been employed. However, access to the IP location in the interaction chamber was difficult and additional systematic errors would have been introduced due to the magnifying optics. Ultimately, LW systems will be prepared without the ability to easily access the IP and suitable methods for determining the IP beam size need to be developed. Therefore, a model of the beam propagation from laser output to focus was created (including the additional $M_{q}^{2}$ introduced by the final focus lens), which took as inputs the laser beam size and $M_{0}^{2}$ and produced as output the focused spot size at the LWIP. To verify the model, measurements were taken of the initial beam size, $M_{0}^{2}$, and focused spot size of a low power $\mathrm{CW}$ laser, and the predicted spot size for these inputs was compared with the measured value. The model is illustrated schematically in Fig. 13.

To find the inputs for the model, a set of laser and lens performance measurements were performed. The $M_{0}^{2}$ of both the high power and $\mathrm{CW}$ lasers were measured by placing a planoconvex lens, $f=1 \mathrm{~m}$ [24] (chosen to avoid introducing any additional aberrations to the measurement) and translating a CCD camera [25] along the optical axis through the focus, measuring the beam size at each longitudinal position. The diagnostic lens was positioned on the table such that it was approximately the same distance from the laser exit port as the final focus lens $(11 \mathrm{~m})$. An

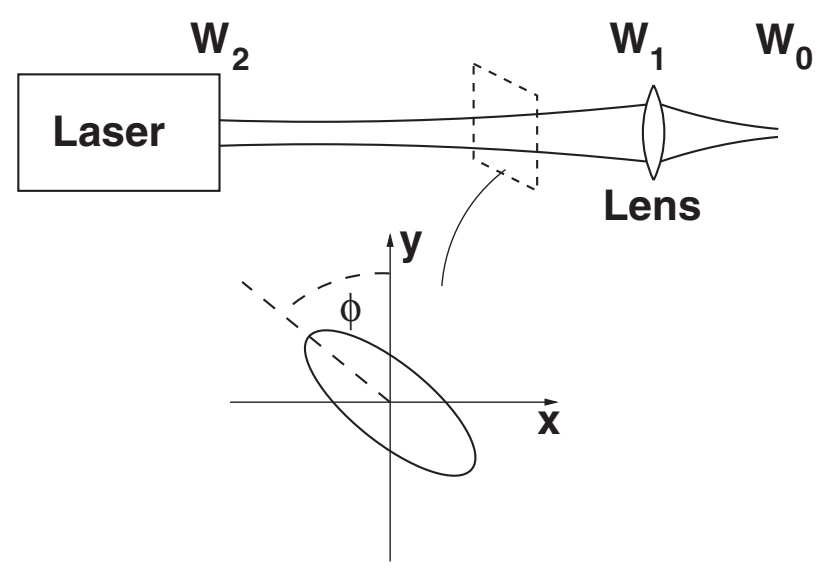

FIG. 13. The locations of the beam size parameters $W$ and the definition of rotation angle $\phi$.

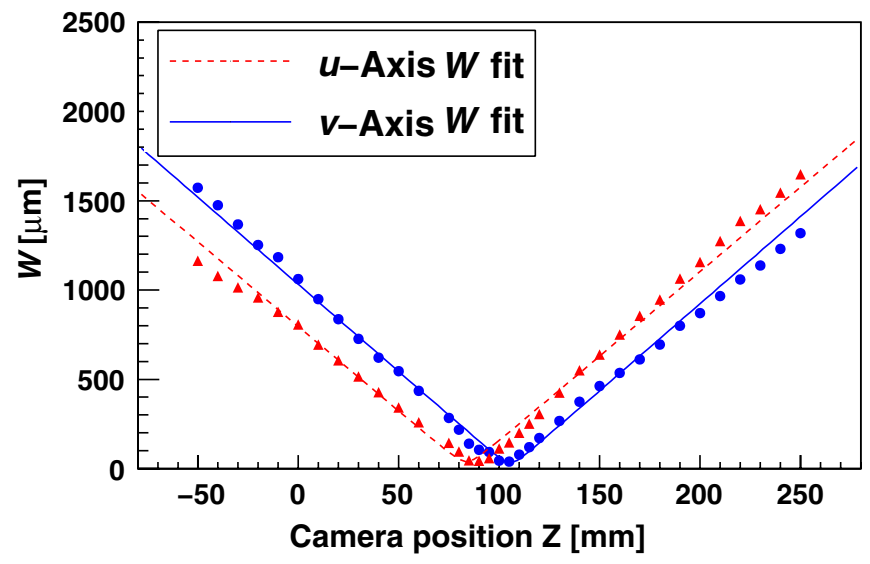

FIG. 14. A profile measurement of the pulsed laser. 
TABLE IV. $\quad M_{0}^{2}$ measurements on the $\mathrm{CW}$ and pulsed lasers. The results are from the fit of Eq. (2) to the laser propagation measurement data.

\begin{tabular}{lcc}
\hline \hline & CW laser & Pulsed laser \\
\hline$M_{0, u}^{2}$ & $1.3 \pm 0.1$ & $2.0 \pm 0.2$ \\
$M_{0, v}^{2}[\mu \mathrm{m}]$ & $1.1 \pm 0.2$ & $1.6 \pm 0.25$ \\
$W_{0, u}[\mu \mathrm{m}]$ & $30 \pm 3$ & $36 \pm 3$ \\
$W_{0, v}[\mu \mathrm{m}]$ & $24 \pm 4$ & $28 \pm 3$ \\
$Z_{0, u}[\mathrm{~mm}]$ & $71.2 \pm 0.1$ & $83.8 \pm 0.1$ \\
$Z_{0, v}[\mathrm{~mm}]$ & $86.7 \pm 0.1$ & $105.4 \pm 0.1$ \\
\hline \hline
\end{tabular}

average of 64 images taken at each position on the optical axis was used to find the size of the beam at that point. Both lasers were simply astigmatic (i.e. the transverse beam profiles were elliptical), so the beam sizes at each point were taken along orthogonal axes, $u$ and $v$ (Fig. 14), where $u$ is defined as the semimajor axis at the input to the lens, and is rotated from the $y$ axis by angle $\phi$ (Fig. 13). A systematic error on each beam size measurement of $\pm 5 \mu \mathrm{m}$ due to the resolution of the camera was added to the statistical error in quadrature. The beam sizes along each axis were fitted to the propagation equation (2), with $W_{0}, z_{0}$, and $M_{0}^{2}$ as fit parameters. The $M_{0}^{2}$ of the pulsed laser was found to be $\sim 2$, whereas for the $\mathrm{CW}$ laser $M_{0}^{2} \sim 1$, a full summary is shown in (Table IV). The propagation model shows that the effect of the astigmatism on the final beam size is small [26].

The input beam sizes of the pulsed and CW lasers on the final focus lens were measured by directing the lasers at a calibrated screen and imaging the screen with a CCD camera. The results of the input beam size measurements are shown in Table $\mathrm{V}$, where $\phi$ is defined as the rotation of the semimajor axis of the transverse beam profile (Fig. 13). The standard deviation in the beam position on the final focus lens was measured using the same camera as $29 \mu \mathrm{m}$. The distance to the final focus lens is $11 \mathrm{~m}$, therefore the standard deviation in the angle from the propagation axis is $2.6 \times 10^{-6} \mathrm{rad}$. With the $56.6 \mathrm{~mm}$ focal length lens this corresponds to a standard deviation in position at the laser focus of $0.15 \mu \mathrm{m}$, which is negligible.

The measurements above gave the transverse mode quality $\left(M_{0}^{2}\right)$ and beam sizes $\left(W_{1}\right)$ on the final focus lens for the pulsed and CW lasers, used as inputs to the simple model described at the beginning of this section. A summary of the measurements (input) and model (output) is shown in Table VI. Rows 1 and 2 show the input beam sizes

TABLE V. Input laser beam sizes $\left(W_{1}\right)$, ellipticities, $\left(\eta_{1}\right)$, and rotation angles $\left(\phi_{1}\right)$.

\begin{tabular}{lrccr}
\hline \hline Laser & $W_{1, u}[\mathrm{~mm}]$ & $W_{1, v}[\mathrm{~mm}]$ & $\eta_{1}$ & $\phi[\mathrm{deg}]$ \\
\hline CW & $9.2 \pm 0.3$ & $9.0 \pm 0.3$ & 0.98 & -13.9 \\
Pulsed & $10.1 \pm 0.3$ & $9.1 \pm 0.3$ & 0.88 & -75.3 \\
\hline \hline
\end{tabular}

of the two lasers, $W_{1}$, in the two axes. In rows 3 and 4 are the estimated beam sizes at the lasers $W_{2}$. These were determined after calculating the divergences by propagating back the distance from the lens to the laser. The divergences were calculated using the locations of the minima of the $1 \mathrm{~m}$ diagnostic lens. The astigmatism angle is from Table V. The error in the measurement of the input beam size dominates the systematic uncertainties in all the outputs of the model because $M_{q}^{2}$ goes as the fourth power in $W_{1}$ (Fig. 9). Lines 6 to 9 in Table VI summarize the measured $M_{0}^{2}$ values, as well as the total $M^{2}$, which includes the contribution $M_{q}^{2}$ from the lens. Lines 10 and 11 in Table VI show the global minimum beam sizes in the two orthogonal axes, $W_{0, u}$ and $W_{0, v}$ of the custom doublet $56.6 \mathrm{~mm}$ focal length lens (Sec. III B) used in laser-wire running, as outputs for both the $\mathrm{CW}$ and pulsed lasers. Lines 12 and 13, $W_{0, x}$ and $W_{0, y}$, are the global minimum beam sizes in the $x$ and $y$ axes, taking into account the astigmatism angle $\phi$.

To test the model predictions, the focused spot size of the $\mathrm{CW}$ laser was also measured directly using a beam profiler [22]. The $\mathrm{CW}$ input beam size on the final focus lens was set to be $\sim 9 \mathrm{~mm}$ and beam size measurements were taken at several positions along the optical axis, in a similar manner to the $M_{0}^{2}$ measurements described above. The beam size was defined as the rms beam size with a cut at $0.3 \%$ of the peak projected intensity which was sufficient to remove any background without removing the tails of the profile. The data were fitted with the laser propagation function, Eq. (2), resulting in estimates of $W_{0}$ and $M^{2}$ (Fig. 15). The measurements of the $M^{2}$ after the final lens and $W_{0}$ for the CW laser were $3.47 \pm 0.79$ and $3.25 \pm$ $0.75 \mu \mathrm{m}$ respectively, in good agreement with the predicted values from the model. This gives us confidence in the main result of the model, which is the estimate for the

TABLE VI. Input/output to the laser propagation model and measured values. Model means the value is either an input to or an output from the model.

\begin{tabular}{lcc}
\hline \hline & $\mathrm{CW}$, model & Pulsed, model \\
\hline$W_{1, u}[\mathrm{~mm}]$ & $8.7-9.3$ (input) & $8.8-9.4$ (input) \\
$W_{1, v}[\mathrm{~mm}]$ & $8.9-9.5$ (input) & $9.8-10.4$ (input) \\
$W_{2, u}[\mathrm{~mm}]$ & $6.5-7.3$ (output) & $5.8-6.2$ (output) \\
$W_{2, v}[\mathrm{~mm}]$ & $6.5-7.2$ (output) & $5.4-5.8$ (output) \\
$\phi[\mathrm{deg}]$ & -13.9 (input) & -75.3 (input) \\
$M_{0, u}^{2}$ & $1.3 \pm 0.1$ (input) & $2.0 \pm 0.2$ (input) \\
$M_{0, v}^{2}$ & $1.100_{-0.1}^{+0.2}$ (input) & $1.6 \pm 0.2$ (input) \\
$M_{u}^{2}$ & $3.07_{-0.60}^{+0.75}$ (output) & $3.53_{-0.64}^{+0.80}$ (output) \\
$M_{v}^{2}$ & $3.26_{-0.68}^{+0.89}$ (output) & $5.25_{-1.1}^{+1.4}$ (output) \\
$W_{0, u}[\mu \mathrm{m}]$ & $3.24_{-0.53}^{+0.67}$ (output) & $3.73_{-0.58}^{+0.69}$ (output) \\
$W_{0, v}[\mu \mathrm{m}]$ & $3.41_{-0.61}^{+0.79}$ (output) & $4.99_{-0.89}^{+1.2}$ (output) \\
$W_{0, x}[\mu \mathrm{m}]$ & $3.41_{-0.61}^{+0.79}$ (output) & $4.04_{-0.58}^{+0.71}$ (output) \\
$W_{0, y}[\mu \mathrm{m}]$ & $3.26_{-0.54}^{+0.68}$ (output) & $5.07_{-0.85}^{+1.1}=2 \sigma_{L 0}$ (output) \\
\hline \hline
\end{tabular}




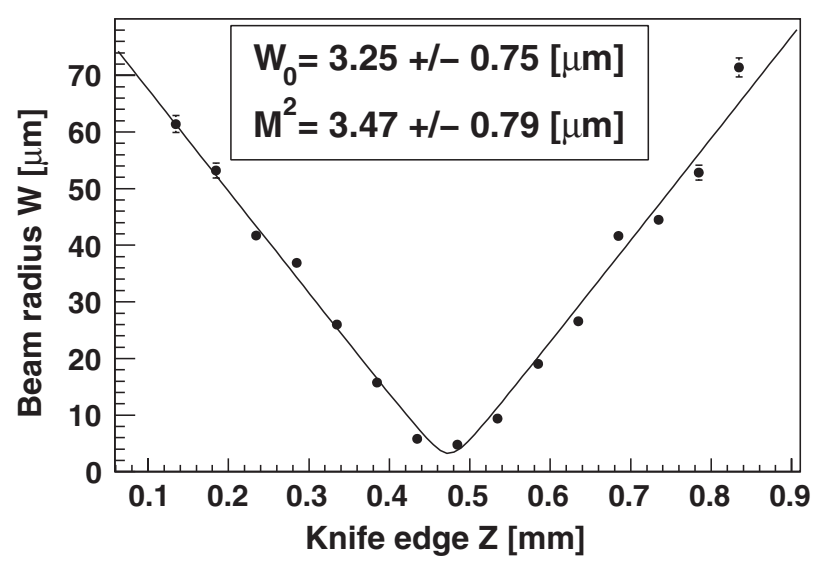

FIG. 15. An IP profile measurement in the horizontal axis of the $\mathrm{CW}$ laser after the custom final focus lens.

beam size of the pulsed laser at the LWIP of $W_{0, y}=$ $5.07_{-0.85}^{+1.1} \mu \mathrm{m}$.

\section{RESULTS}

In this section collision data from a single $16 \mathrm{~h}$ shift, beginning on 29 May 2008, are presented. The data taking was organized in the following stages: (1) Find beam overlap and collisions between laser pulse and electron beam. (2) Perform a laser waist scan to find the focus position along the optical axis. (3) Perform a single quadrupole scan to find the smallest electron beam size. (4) Perform a skew quadrupole scan to minimize vertical electron beam size. (5) Once the laser timing and focus position and electron beam size have been optimized, take a detailed laser-wire scan of the smallest electron beam.

Before taking laser-wire data, the laser was tuned to high energy, ( $400 \mathrm{~mJ}$ pulse energy), which remained steady for the entire shift. Then the laser was aligned and input beam size measurements were performed as described in Sec. IV. The laser-wire electron beam optics as presented in Sec. II were then loaded and the orbit in the extraction line was tuned to minimize the background in the Cherenkov detector. The dispersion measurements presented in Sec. II A were then performed before beginning the beam overlap procedure.

\section{A. Beam overlap procedure}

The procedure to overlap the beams was as follows: (1) Determine the laser position in the interaction chamber using the screen and the CW laser. (2) Set coarse timing and spatial overlap using the screen. (3) Fine-tune the temporal overlap by performing a timing system $357 \mathrm{MHz}$ rf phase scan. (4) Perform a vertical beam position scan to find the collision signal. (5) Perform vertical scans at different lens focus positions in order to focus the laser onto the electron beam.

First, the CW laser was used with the screen to determine the position of the laser focus within the interaction chamber. The screen was then moved a few microns above this point. The pulsed laser was operated at low power by disabling the final amplification stage, and the screen was brought close to the electron beam by moving the chamber vertically. This generated an optical diffraction radiation (ODR) or optical transition radiation (OTR) pulse in the direction of the laser beam which passed through the laser optics to the avalanche photodiode (APD). It is entirely possible that the light from the screen included incoherent synchrotron radiation (ISR) that was reflected from the screen towards the APD, but ISR arrives at the screen at the same time as the electron pulse. Heavily attenuated pulses from the main laser were also incident on the APD. As shown in Fig. 5, the attenuated laser light passed underneath the screen close to the point where the ODR/ OTR light was produced. Therefore the path lengths to the APD were very similar, and certainly differed by much less than the laser pulse duration of $168 \pm 0.5 \mathrm{ps}$, or pulse length of $5.02 \pm 0.02 \mathrm{~cm}$. The difference in arrival times of the laser pulse and beam induced radiation could therefore be monitored directly by observing the signal from the APD on a fast scope [27]. The laser timing was modified so that the laser light arrival at the APD was as close as possible to the arrival of light from the screen, typically much less than an rf bucket of $2.8 \mathrm{~ns}$, and less than the laser pulse duration of $168 \pm 0.5$ ps. The method of using a single detector removes any possible systematic offset in the timing of the two pulses, as the timing is measured with exactly the same path difference from the LWIP and same detector and cable. In order to obtain spatial overlap the beams were configured so that the electron beam passed just below the screen and the laser beam was obscured. The laser beam was moved vertically downwards until a laser signal was observed on the APD. This guaranteed that both beams were just below the height defined by the knife edge. The ODR/OTR screen was then removed and the laser returned to full power. This process typically resulted in a Compton signal observable over the detector background, as seen on a scope connected to the output of the Compton detectors. The signal was then transferred to a CAMAC

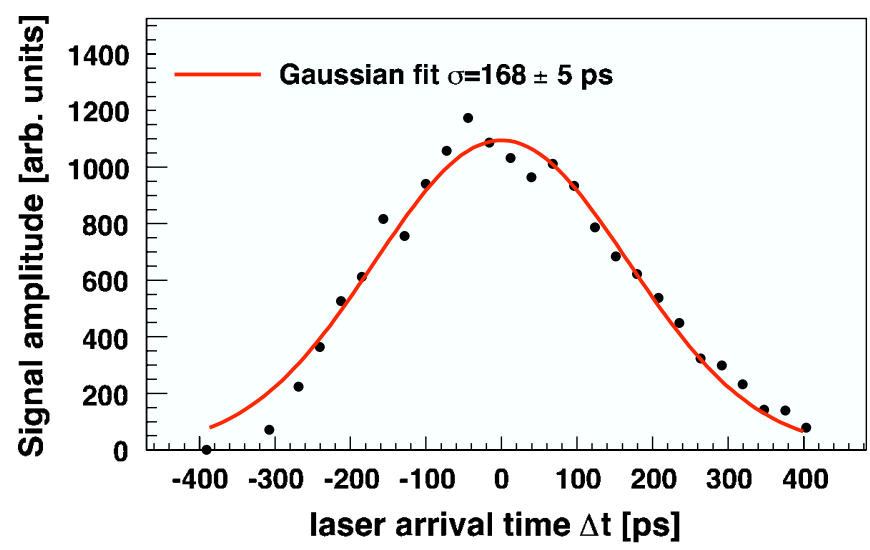

FIG. 16. Compton signal maximum amplitude as a function of laser pulse arrival time. 
based digitizer system. The Compton signal was optimized by varying the phase of the $357 \mathrm{MHz}$ laser locking rf signal (which changed the arrival time of the laser pulse), an example of which is shown in Fig. 16. The fit to the plot in Fig. 16 is a normal distribution with a width of $168 \pm$ $5 \mathrm{ps}$, in reasonable agreement with the expectations for the laser [28]. Having maximized the temporal overlap, the vertical and laser waist position could be optimized for maximum signal.

\section{B. Collision measurements}

In the collision data presented below, various methods of analyzing the convoluted electron/laser beam profiles in order to extract the width of the profile are considered. The conditions for validity and usefulness of these methods are discussed in Secs. V B 4 and V B 6.

First, the background is subtracted by fitting a straight line function to the points at the edges of the distribution and then subtracting this function from all points in the distribution. The width is then calculated in one of the following ways: (1) The rms of the distribution is calculated after applying cut at $0.3 \%$ of the peak intensity in order to remove the background noise, which was the same cut applied to the laser and lens model and measurements. (2) A Gaussian plus a constant is fit to the data.

\section{Laser waist scan}

Laser waist scans were performed in the following way: the laser focus position was changed by moving the interaction chamber and lens along the laser propagation axis and at each focus position a vertical scan was performed. $\sigma_{s}$ was plotted as a function of lens position and fitted to the laser propagation function [Eq. (2)]. Through this process, the laser beam focus position could be found.

\section{Single quadrupole scan}

The electron beam focus position can be changed by varying the current through an upstream quadrupole

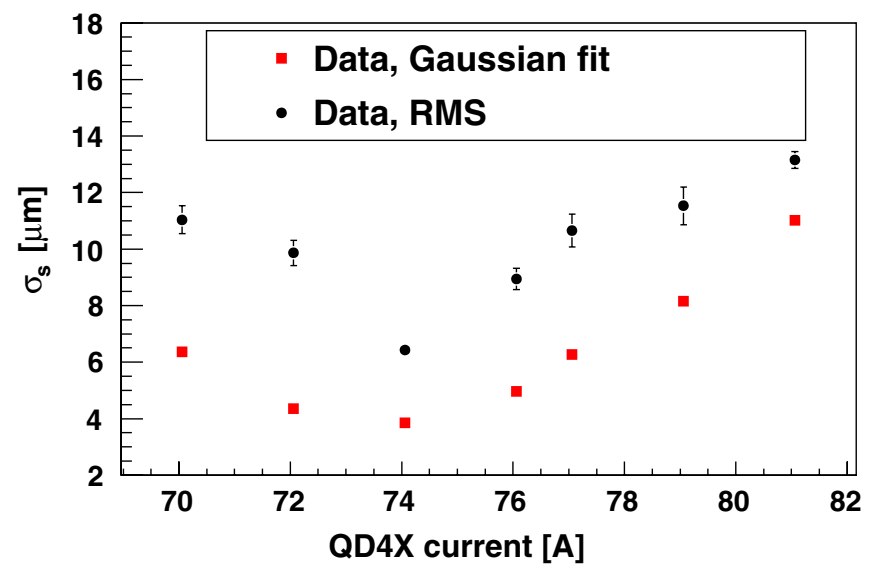

FIG. 17. QD4X quadrupole scan. QF4X current was kept constant at $73.58 \mathrm{~A}$.
(QD4X) to change its focusing strength. Quadrupole focusing strength $l k_{\mathrm{QF} 4 \mathrm{X}}$ was also changed in order to move the horizontal axis focus position at the same rate. A quadrupole scan was performed by carrying out a laserwire vertical chamber scan at each different quadrupole setting. The current through QD4X was changed while the current through QF4X was held constant at 73.58 A and the results are shown in Fig. 17.

\section{Skew quadrupole scan}

The QD4X current was set to the current which gave the minimum beam size, at $74.06 \mathrm{~A}$, and skew quadrupole QS1X was scanned to try and reduce the vertical beam size (Fig. 18). Changing the skew quadrupoles could have changed the focus position slightly. A further quad scan could have confirmed this but due to time constraints was not possible.

\section{Smallest $\sigma_{s}$ laser-wire scan}

A plot of the smallest rms $\sigma_{s}$ laser scan is shown in Fig. 19. This scan was performed after fully optimizing the experiment as described above, with the QD4X current and 76.04 A and the QS1X current at $-2 \mathrm{~A}$. The smallest rms convoluted beam size, $\sigma_{s}$, was measured as $5.4 \pm 0.1 \mu \mathrm{m}$. In Sec. III A the laser beam size was estimated to be $\sigma_{L 0}=$ $2.54_{-0.43}^{+0.55} \mu \mathrm{m}$ (Table VI). Therefore the minimum measured rms electron beam size was $4.8 \pm 0.3 \mu \mathrm{m}$. The contribution to the vertical electron beam size due to dispersion was found to be $3.03 \pm 0.66 \mu \mathrm{m}$. These results are consistent with an electron beam size due to the beta function of $3.7 \pm 0.7 \mu \mathrm{m}$. The results, along with the results from the Gaussian fit for comparison, are summarized in Table VII. Typically, as can be seen from Figs. 17 and 18 and Table VII, the Gaussian fit yields a smaller value for $\sigma_{s}$ indicating some non-Gaussian shape.

Figure 19 also shows the laser wire fitted with a Gaussian, as expected from the theory presented in

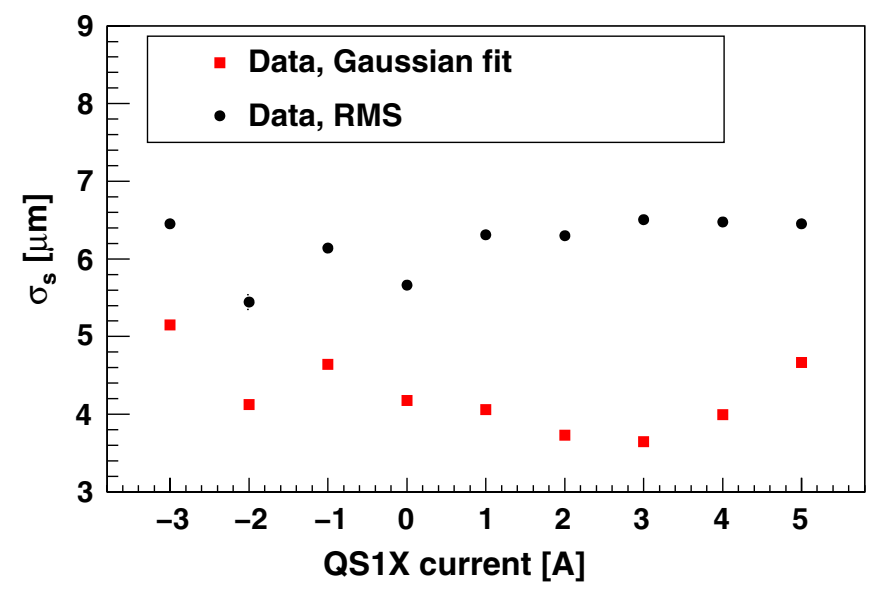

FIG. 18. QS1X skew quad vs $\sigma_{s}$ scan. QD4X was set to $74.06 \mathrm{~A}$. 


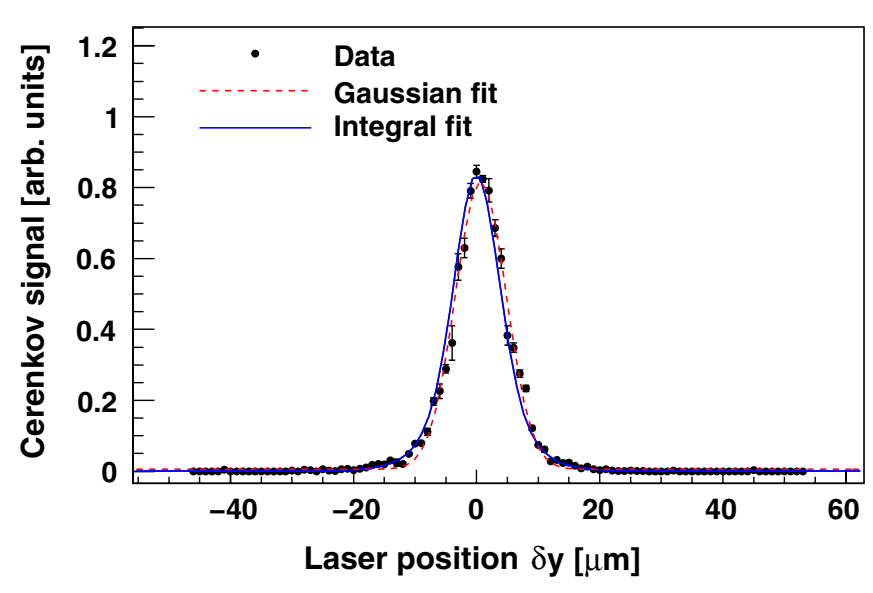

FIG. 19. The smallest rms $\sigma_{s}$ laser-wire scan measured using the laser-wire system.

Sec. I B. However, this analysis assumes that the Rayleigh range of the laser is longer than the horizontal size of the electron beam. When this is not the case the wings of the smaller laser-wire profiles are enhanced and are not well fitted to a Gaussian. It is possible to numerically solve the generalized function Eq. (6) (this fit is also shown in Fig. 19) and this method is discussed in Sec. V B 6, but as we show, to correctly extract the vertical electron beam size using this method requires the horizontal electron beam size, which was unknown in our experiment. Therefore the rms method seems the most reliable. However, Fig. 18 and the results in Sec. V B 6 imply that the fit function methods have more sensitivity to the electron beam size at rms $\sigma_{s}$ below about $5 \mu \mathrm{m}$. This could be because the enhanced profile wings at these beam sizes, which contribute to rms $\sigma_{s}$, are due to the Rayleigh range effects described in Sec. V B 6 and not the electron beam profile.

\section{Laser propagation collision data}

The electron beam size found in the previous section (Sec. V B 4) was checked for consistency with the laser waist scan (Sec. V B 1). If the extracted electron beam size is correct then the data for the laser waist scan should be consistent with the laser measurements and model presented in Sec. IV. The size of the electron beam was not precisely known when this data was taken, but subtracting

TABLE VII. $\sigma_{s}$ calculated from Fig. 19 using a Gauss + const fit and an rms calculation. $\sigma_{s}$ is the signal sigma. The integral fit result is not shown because the horizontal beam size was unknown and therefore the vertical beam size could not be calculated (Sec. V B 6).

\begin{tabular}{lcr}
\hline \hline Analysis method & $\sigma_{s}[\mu \mathrm{m}]$ & $\sigma_{e}[\mu \mathrm{m}]$ \\
\hline Gauss + const & $4.126 \pm 0.001$ & $3.25 \pm 0.4 \mu \mathrm{m}$ \\
$\mathrm{rms}$ & $5.4 \pm 0.1$ & $4.8 \pm 0.3 \mu \mathrm{m}$ \\
\hline \hline
\end{tabular}

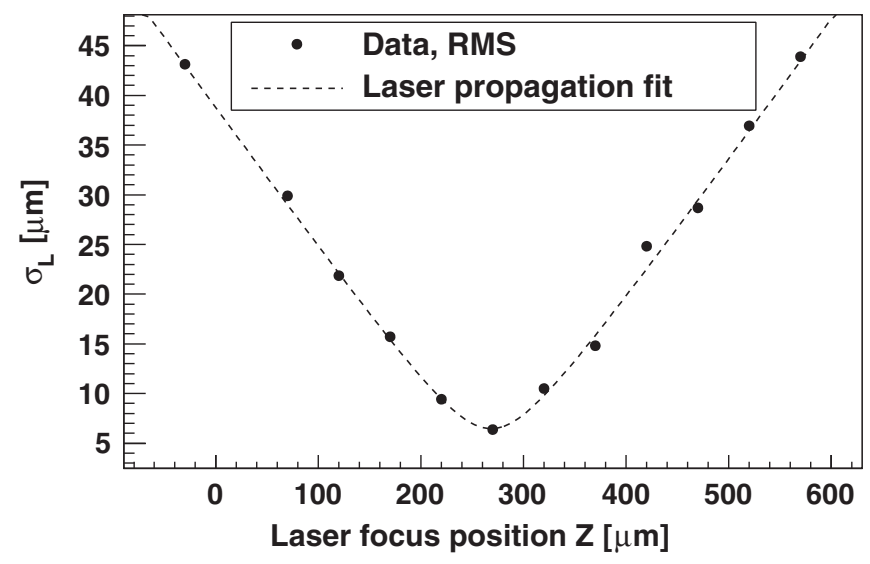

FIG. 20. Waist scan. The laser focus position is $x$ in electron beam coordinates. The fit function is the laser propagation function, Eq. (2).

the smallest electron beam size in quadrature from each rms $\sigma_{s}$ data point gives an upper limit to the laser beam size at that point. These upper laser beam sizes are plotted in Fig. 20, and the results from the laser propagation fit function are shown in Table VIII. These data are completely consistent with the results of the laser propagation model given in Table VI.

\section{Integral fit to collision data}

As discussed in Sec. VB 4, the Gaussian fit expected from Eq. (7) is not a good fit to the laser-wire convoluted profile data when Rayleigh range effects are appreciable. This can be seen in Fig. 19, where the Gaussian fit to the wings of the profile is not good, and more clearly in Fig. 21, which shows a laser-wire scan taken with QS1X at 3 A. At this QS1X setting the Gaussian fit $\sigma_{s}$ is a minimum, although the fit to this data is poor in the wings of the profile.

It is, however, possible to evaluate the number of Compton scattered photons without the assumption that the Rayleigh range of the laser focus is large compared with the electron beam size. This yields [10] an integral fit function [Eq. (11)] which depends on the horizontal and vertical electron beam sizes. This function includes nonGaussian tails when the horizontal electron beam size is similar to the Rayleigh range of the laser. It is given by

$$
\begin{aligned}
\left\langle N_{\gamma}\right\rangle & \propto\left\langle S_{\gamma}\right\rangle(\delta x, \delta y) \\
& =\frac{I_{l} I_{e}}{2 \pi \sigma_{e x}} \int \frac{d x}{\sigma_{s}(x, \delta x)} \exp \left[-\frac{x^{2}}{2 \sigma_{e x}^{2}}-\frac{\delta y^{2}}{2 \sigma_{s}^{2}(x, \delta x)}\right],
\end{aligned}
$$

TABLE VIII. Results of laser propagation fits to Fig. 20.

\begin{tabular}{lcc}
\hline \hline Analysis method & $W_{0}[\mu \mathrm{m}]$ & $M^{2}$ \\
\hline $\mathrm{rms}$ & $<6.5$ & $<5.4$ \\
\hline \hline
\end{tabular}




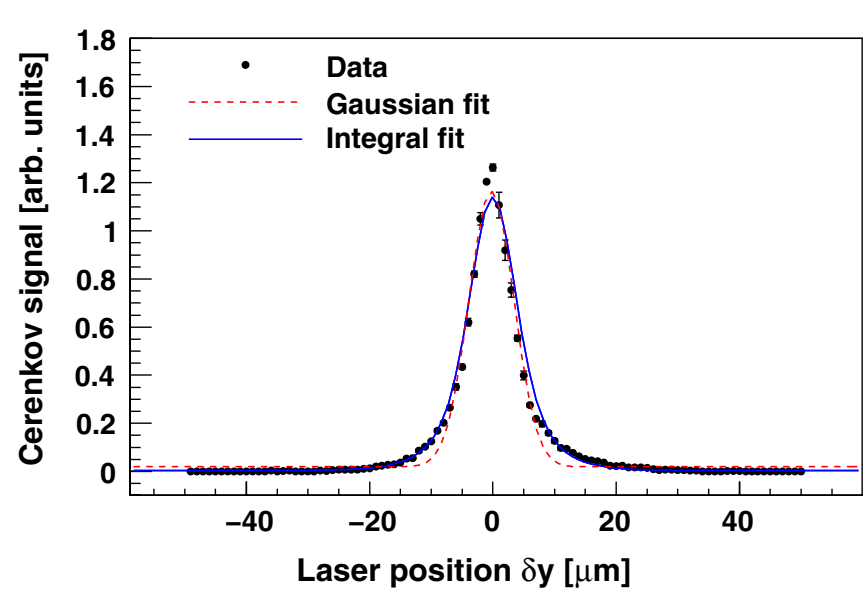

FIG. 21. The smallest $\sigma_{s}$ laser scan performed using the laserwire system, according to both a Gaussian and full integral fit. The integral fit function [Eq. (11)] is shown.

where $I_{l}$ and $I_{e}$ are constants related to the laser and electron beam intensities, and

$$
\sigma_{s}(x, \delta x)=\sqrt{\sigma_{e y}^{2}+\sigma_{L}^{2} f_{R}(x-\delta x)},
$$

where $\delta x$ and $\delta y$ are the horizontal and vertical displacements, respectively, $\sigma_{e x}$ and $\sigma_{e y}$ are the horizontal and vertical transverse electron beam sizes, and $f_{R}(x)$ is given by

$$
f_{R}(x)=1+\left(\frac{x}{x_{R}}\right)^{2},
$$

where $x_{R}$ is the Rayleigh range.

Figures 19 and 21 show the integral fit to the data with a ratio of the horizontal to vertical electron beam size of 50 . It can be seen from the figures that the integral fit is a better fit to the data than a Gaussian, and this is confirmed by the fact that the rms of the data in Fig. 21 is $\sigma_{s}=6.30 \pm$ $0.04 \mu \mathrm{m}$ and the rms of the fit function is $\sigma_{s}=6.34 \mu \mathrm{m}$ i.e. they are practically the same. Once the ratio $\sigma_{e x} / \sigma_{e y}$ is set, the only free parameter of the integral fit, apart from the centroid and amplitude, is the electron beam vertical size $\sigma_{e}$. The other parameters are fixed to the values in Table IX which are taken from the mean measurements in Table VI. The input beam size versus $M^{2}$ model is used to calculate the laser beam size $\sigma_{L 0}$ in the fit function.

Table X summarizes $\sigma_{s}$ and $\sigma_{e y}$ for the data in Figs. 19 and 21 calculated from the rms of the data, a Gaussian fit and for the integral fit with various values of the electron beam aspect ratio. The errors include statistical errors due to the fitting or rms calculation and systematic errors from the uncertainty in $\sigma_{L 0}$. It can be seen from this table that the values of $\sigma_{e y}$ vary by a factor of nearly 4 between the rms analysis and the integral fit for large aspect ratios of the beam. This is predominantly because using the rms method attributes the enhancement of the wings of the laser-wire profile to the electron beam size, whereas it is accounted
TABLE IX. Fixed parameters of the integral fit.

\begin{tabular}{lc}
\hline \hline Parameter & Value \\
\hline$\sigma_{L 0}[\mu \mathrm{m}]$ & 2.54 \\
Focal length of lens $f[\mathrm{~mm}]$ & 56.6 \\
\hline \hline
\end{tabular}

TABLE X. Top: $\sigma_{s}$ using rms and Gaussian fit and $\sigma_{e y}$ using integral fit calculated from Fig. 21. Where two errors are shown, the first is a systematic error due to the uncertainty in $\sigma_{L 0}$ and the second is the statistical uncertainty of the fit or rms calculation. Otherwise, the error is the statistical error. Bottom: same from Fig. 19.

\begin{tabular}{lcc}
\hline \hline Analysis method & $\sigma_{s}[\mu \mathrm{m}]$ & $\sigma_{e y}[\mu \mathrm{m}]$ \\
\hline Gauss + const & $3.65 \pm 0.08$ & $2.62_{-0.68}^{+0.36} \pm 0.11$ \\
rms & $6.50 \pm 0.08$ & $5.98_{-0.26}^{+0.16} \pm 0.09$ \\
Integral fit, $\sigma_{e x} / \sigma_{e y}=30$ & $3.38_{-0.43}^{+0.48} \pm 0.01$ & $2.22_{+0.09}^{-0.16} \pm 0.01$ \\
Integral fit, $\sigma_{e x} / \sigma_{e y}=40$ & $3.12_{-0.44}^{+0.51} \pm 0.01$ & $1.81_{-0.15}^{+0.10} \pm 0.01$ \\
Integral fit, $\sigma_{e x} / \sigma_{e y}=50$ & $2.96_{-0.44}^{+0.53} \pm 0.01$ & $1.52_{-0.14}^{+0.10} \pm 0.01$ \\
Gauss + const & $4.13 \pm 0.06$ & $3.25_{-0.52}^{+0.29} \pm 0.08$ \\
rms & $5.40 \pm 0.11$ & $4.77_{-0.3}^{+0.2} \pm 0.12$ \\
Integral fit, $\sigma_{e x} / \sigma_{e y}=30$ & $3.21_{-0.40}^{+0.45} \pm 0.01$ & $1.97_{-0.11}^{+0.00} \pm 0.01$ \\
Integral fit, $\sigma_{e x} / \sigma_{e y}=40$ & $3.00_{-0.43}^{+0.49} \pm 0.01$ & $1.59_{-0.12}^{+0.03} \pm 0.01$ \\
Integral fit, $\sigma_{e x} / \sigma_{e y}=50$ & $2.86_{-0.43}^{+0.51} \pm 0.00$ & $1.32_{-0.10}^{+0.04} \pm 0.01$ \\
\hline \hline
\end{tabular}

for by the diffractive spread of the laser beam in the integral fit. This suggests strongly that the profile width as measured by calculating the rms of the data may be an overestimate when Rayleigh range effects are important. However, the beam size extracted from the integral fit is strongly dependent on the aspect ratio. This is illustrated in Figs. 22 and 23, which show the beam size and the $\chi^{2} / N D F$ of the overlap fit for different values of this ratio. The quality of the fit does not significantly improve for values of $\sigma_{e x} / \sigma_{e y}>100$ but Fig. 22 demonstrates that

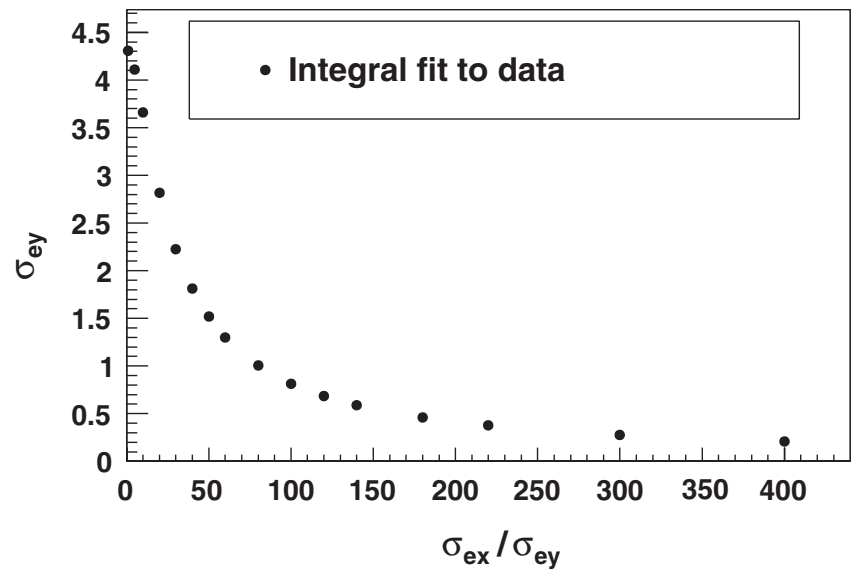

FIG. 22. Fit results of Fig. 21 as a function of the $x-y$ electron beam aspect ratio. 


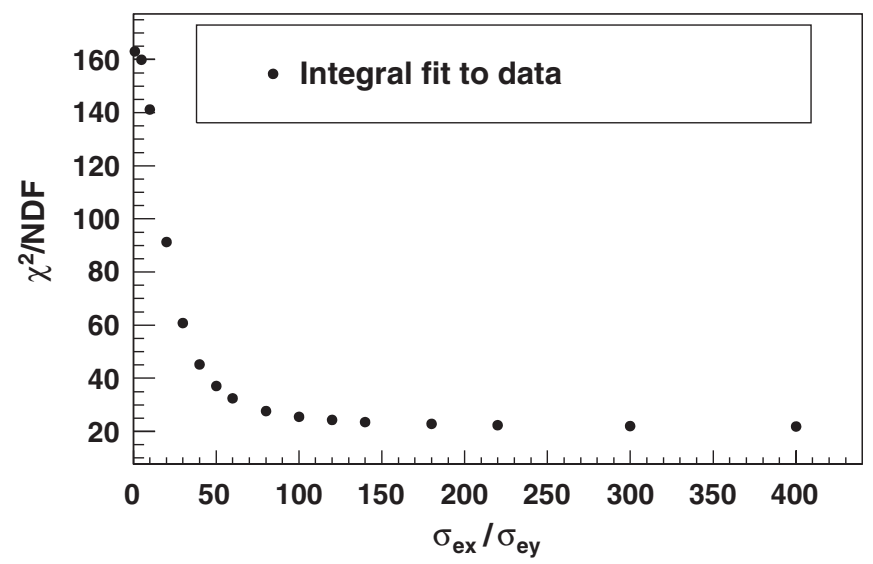

FIG. 23. $\chi^{2} / N D F$ of the fit to Fig. 21 for different values of $\sigma_{e x} / \sigma_{e}$.

without a proper estimate of the horizontal electron beam size it is not really possible to use the integral fit to find the vertical beam size, which is why we have chosen to use the rms value for the data presented in the paper. One of the improvements of the laser-wire experiment is to make it a 2D system, which would avoid this problem in the future. Furthermore, the new laser-wire installation at the ATF2 beam line incorporates an OTR system located at the laserwire IP, which will give independent horizontal and vertical beam size measurements.

\section{Emittance measurement using the laser wire}

Data from a quadrupole scan fit (Fig. 24), dispersion measurement (Sec. II A), and laser profiling (Sec. III A) are combined to produce an emittance measurement. $\sigma_{s}$ is the quadrature sum of the electron beam size due to the beta function $\sigma_{\mathrm{e}, \beta}$, the electron beam size due to dispersion $\sigma_{\mathrm{e}, \eta}$, and the laser beam size at the laser waist $\sigma_{\mathrm{L} 0}$. The contribution to $\sigma_{s}$ from the electron beam size due to dispersion together with the laser is given by $\sigma_{\eta+\mathrm{L}}=$ $\sqrt{\sigma_{\mathrm{e}, \eta}^{2}+\sigma_{\mathrm{L} 0}^{2}}=\sqrt{3.03^{2}+2.54^{2}} \mu \mathrm{m}=3.95 \mu \mathrm{m}$. This value of $\sigma_{e, \eta}$ comes from the dispersion measurements (Sec. II A) and $\sigma_{\mathrm{L} 0}$ is from the laser measurements (Sec. IV). The error propagation is calculated in the following way. The contributions to the size due to dispersion and laser wire add in quadrature. Then the error is given by

$$
\begin{gathered}
(\delta \sigma)_{\eta+\mathrm{L}}^{2}=\left(\delta \sigma_{e, \eta} \frac{\partial \sigma_{\eta+\mathrm{L}}}{\partial \sigma_{e, \eta}}\right)^{2}+\left(\delta \sigma_{\mathrm{L} 0} \frac{\partial \sigma_{\eta+\mathrm{L}}}{\partial \sigma_{\mathrm{L} 0}}\right)^{2} \\
=\left[\left(\delta \sigma_{e, \eta}\right)^{2} \sigma_{e, \eta}^{2}+\left(\delta \sigma_{\mathrm{L} 0}\right)^{2} \sigma_{\mathrm{L} 0}^{2}\right] \frac{1}{\sigma_{\eta+\mathrm{L}}^{2}},
\end{gathered}
$$

where $\delta x$ means the error on the measurement of $x$. From the results $\sigma_{\mathrm{L} 0}=2.54 \pm 0.50 \mu \mathrm{m}$ and $\sigma_{e, \eta}=$ $3.03 \pm 0.66 \mu \mathrm{m}$, using Eq. (15) we find that

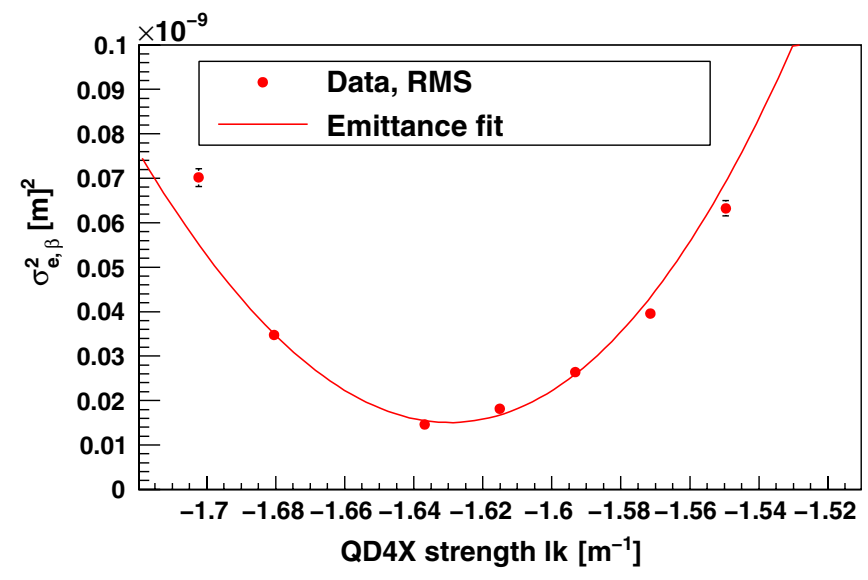

FIG. 24. Laser wire measured $\sigma_{e}^{2}$ vs QD4X $1 \mathrm{k}$ value.

$$
\begin{gathered}
(\delta \sigma)_{\eta+\mathrm{L}}^{2}=\left(0.66^{2} \times 3.03^{2}+0.50^{2} \times 2.54^{2}\right) \frac{1}{3.95^{2}} \\
\delta \sigma_{\eta+\mathrm{L}}=0.60 \mu \mathrm{m}
\end{gathered}
$$

so $\sigma_{\eta+\mathrm{L}}=4.0 \pm 0.6 \mu \mathrm{m}$.

An emittance measurement was carried out by performing a two quadrupole (QD4X and QF4X) scan and fitting a parabola to the squared beam size as a function of quadrupole strength (Fig. 24). Subtracting $\sigma_{\eta+\mathrm{L}}$ in quadrature from each beam size measurement in the quadrupole scan, the emittance was measured as $560_{-110}^{+70} \mathrm{pm}$, taking into account the above error on $\sigma_{\eta+\mathrm{L}}$. The upper and lower values of $\epsilon$ come from subtracting the lower and upper values of $\sigma_{\eta+\mathrm{L}}$ in quadrature from each beam size measurement before fitting. As with the wire scanner emittance measurement of $207 \mathrm{pm}$ in the previous month, this value is of the order of 10 times the nominal value needed to produce a $1 \mu \mathrm{m}$ vertical electron beam size at the LWIP. It is important to note that the entire quadrupole scan shown in Fig. 24 used laser-wire scans with $\sigma_{s}$ below $10 \mu \mathrm{m}$, possibly explaining the large uncertainty and the systematic shift in the central value compared to the traditional wire measurement.

\section{CONCLUSIONS}

We have constructed and operated a laser-wire transverse beam size measurement system at the ATF, which successfully measured electron beam sizes between approximately 40 and $4.8 \mu \mathrm{m}$. The upper limit is dictated by the signal to noise for the Compton detector at the $150 \mathrm{MW}$ peak laser power used for collision. An increase in laser power of a factor of 4 can be easily delivered from the laser, enabling the measurement of rather large beam sizes of $80 \mu \mathrm{m}$, without any improvement of the Compton detection system. The lower limit was set by the laser and optical system, and by the emittance of the ATF electron beam, which was not optimal (Sec. II A). 
The Cherenkov signal is background subtracted using data points outside of the Compton signal region. Beam sizes are then calculated using the rms of the distribution, which gives a meaningful measurement for any profile shape. First, a cut is made at $0.3 \%$ intensity to remove background noise, in exactly the same way as with the laser and lens model and measurements. These data were also fitted to a Gaussian function for comparison. Rayleigh range effects could contribute when the beam is small (Sec. V B 6). The full integral fit to collision data is preferable (Sec. VB 6) but this was not used in the main analysis because there was no precise knowledge of the horizontal electron beam size.

Measurements were made to account for contributions to $\sigma_{s}$, the size of the convolution between the electron beam and laser beam. Contributions to the electron beam size are from the emittance, and from the dispersion. Factors contributing to the laser beam size include the input beam size on the lens, and the $M^{2}$ laser quality factor. Simulations and measurements indicate that the lens introduces spherical aberrations above a certain input beam size (Secs. and IV) and these affect the quality of the laser; an input laser beam with $M_{0}^{2}=1$ may have a greater $M^{2}$ downstream of the lens. The dominant error in the estimation of the final focus laser beam size, $\sigma_{L 0}$, comes from the error in the measurement of the input beam size. Simulations and measurements predict that $\sigma_{L 0}<1 \mu \mathrm{m}$ could be achieved by reducing the input beam size (Fig. 9). The maximum contribution to the beam size from a simple astigmatism is small $(<0.1 \mu \mathrm{m})$.

The smallest convoluted beam size, $\sigma_{s}$, was measured after skew quadrupole tuning as $5.4 \pm 0.1 \mu \mathrm{m}$ (Table VII and Fig. 19). In Sec. III A the laser beam size was estimated to be $\sigma_{L 0}=2.54_{-0.43}^{+0.55} \mu \mathrm{m}$ (Table VI). Therefore the minimum measured rms electron beam size was $4.8 \pm 0.3 \mu \mathrm{m}$. The contribution to the vertical electron beam size due to dispersion was found to be $3.03 \pm 0.66 \mu \mathrm{m}$. These results are consistent with an electron beam size due to the beta function of $3.7 \pm 0.7 \mu \mathrm{m}$.

Using all the above information, the electron beam emittance in the ATF extraction line was measured as $560_{-110}^{+70} \mathrm{pm}$ by the laser wire. The design value of $\epsilon_{y}$ for ATF is $20 \mathrm{pm}$ in the damping ring, and this increases in the extraction line to $\sim 3$ times this value, but a value of $207 \mathrm{pm}$ was measured in the previous month using a wire scanner. For a more accurate emittance measurement using the laser wire, a higher resolution dispersion measurement is needed. However, the $\epsilon \sim 560 \mathrm{pm}$ laser-wire measurement and the $207 \mathrm{pm}$ wire scanner measurement are approximately 10 times the nominal value (measured in 2005 , before collisions) needed to produce a $1 \mu \mathrm{m}$ vertical electron beam size.

In the future these measurements should be performed with an input laser beam size of $W_{1}=6 \mathrm{~mm}$ (Fig. 8), which will allow us to achieve an even smaller spot size.
High power fiber lasers are being developed [29] which should be able to produce high power, high quality beams.

To verify that the remaining contribution to $\sigma_{s}$ is due to a large emittance electron beam may require an independent emittance measurement simultaneously with the laser-wire emittance measurement, along with dispersion measurements, during a single data taking shift. The extracted emittance at ATF2 [30] is expected to be smaller and more stable.

The tails in the convoluted distributions could be due to Rayleigh range effects or lens aberration effects, which give similar signal curves, depending on the aspect ratio of the electron beam. The aspect ratio cannot be determined from the fit, so in the future the horizontal electron beam size must be measured independently. Figure 22 suggests that the profile width as measured by calculating the rms of the distribution instead of using the full integral fit function [Eq. (11)] may be an overestimate at the smallest beam sizes, where Raleigh range effects come into play.

\section{ACKNOWLEDGMENTS}

We thank Richard Bingham, Chafik Dirouichi, Thorsten Kamps, and Ian Ross for their contributions early in this project. We are grateful to the KEK ATF group for fruitful discussions and expertise and ATF operators for providing stable beam operations. This work was supported by STFC via the LCABD collaboration, by the Commission of European Communities under the 6th Framework program structuring the European Research area RIDS-011899, and under the FP7 Research Infrastructures project EuCARD, Grant Agreement No. 227579, by the Japanese Society for the Promotion of Science, the Royal Society and The Daiwa foundation.

[1] International Linear Collider Reference Design Report 2007, edited by N. Phinney, N. Toge, and N. Walker, 2007.

[2] P. Karataev et al., Phys. Rev. Lett. 93, 244802 (2004).

[3] P. Tenenbaum and T. Shintake, Annu. Rev. Nucl. Part. Sci. 49, 125 (1999).

[4] R. Alley et al., Nucl. Instrum. Methods Phys. Res., Sect. A 379, 363 (1996).

[5] M. Ross, in Proceedings of the 20th Particle Accelerator Conference, Portland, OR, 2003 (IEEE, New York, 2003), pp. 503-507.

[6] Y. Honda et al., Nucl. Instrum. Methods Phys. Res., Sect. A 538, 100 (2005).

[7] A. Bosco et al., Nucl. Instrum. Methods Phys. Res., Sect. A 592, 162 (2008).

[8] T. Shintake, Nucl. Instrum. Methods Phys. Res., Sect. A 311, 453 (1992).

[9] S. Assadi, in Proceedings of the 10th European Particle Accelerator Conference, Edinburgh, Scotland, 2006 
(EPS-AG, Edinburgh, Scotland, 2006), pp. 3161-3163, THPCH156.

[10] I. Agapov, G. A. Blair, and M. Woodley, Phys. Rev. ST Accel. Beams 10, 112801 (2007).

[11] A.E. Siegman, Lasers (University Science Books, Mill Valley, CA, 1986).

[12] A. E. Siegman, Appl. Opt. 32, 5893 (1993).

[13] J. A. Ruff and A. E. Siegman, Opt. Quantum Electron. 26, 629 (1994).

[14] A. Bosco, S. T. Boogert, G. E. Boorman, and G. A. Blair, Appl. Phys. Lett. 94, 211104 (2009).

[15] F. Hinode et al., ATF Accelerator Test Facility Design and Study Report No. 4, KEK, Tsukuba, Japan, 1995 [http://lcdev.kek.jp/ATF/Pub/KEK-I-95-4.pdf].

[16] F. C. Iselin, in Proceedings of the 12th International Conference on High Energy Accelerators, Batavia, IL, 1983 (Fermilab, Batavia, IL, 1983).

[17] F. C. Iselin, Report No. CERN/LEP-TH/85-15.

[18] H. Grote and F.C. Iselin, in Proceedings of the International Conference on Accelerator and Large Experimental Physics Control Systems, Vancouver, Canada, 1989 (Elsevier Science, New York, 1990).
[19] A. W. Chao and M. Tigner, Handbook of Accelerator Physics and Engineering (World Scientific, Singapore, 2006).

[20] K. Kubo and T. Okugi, ATF Technical Report No. 19, KEK, Tsukuba, Japan, 1997 [http://atfweb.kek.jp/atf/ Reports/ATF-97-19.pdf].

[21] Zemax: software for optical design [http://www.zemax .com].

[22] DataRay BeamMap-C [http://www.dataray.com].

[23] I. Agapov, G. A. Blair, S. Malton, and L. Deacon, Nucl. Instrum. Methods Phys. Res., Sect. A 606, 708 (2009).

[24] CVI PLCX-50.8-515.1-C [http://www.cvilaser.com].

[25] DataRay WinCam-D [http://www.dataray.com].

[26] L. Deacon, Ph.D. in Physics, Royal Holloway, University of London, 2009.

[27] Tektronix TDS-684C, 2.5 Gigasamples/second, $300 \mathrm{MHz}$ input bandwidth [http://www.tek.com].

[28] Coherent Inc. Japan (private communication).

[29] L. Corner et al., in Proceedings of the 11th European Particle Accelerator Conference, Genoa, 2008 (EPS-AG, Genoa, Italy, 2008), pp. 1359-1361, TUPC124.

[30] B. I. Grishanov et al., (ATF2)SLAC-R-771. 